\title{
Mechanism of Permselectivity of Porous Polymeric Membranes in Ultrafiltration Process
}

\author{
Kenji KAMIDE and Sei-ichi ManABE \\ Textile Research Laboratory, Asahi Chemical Industry Co., Ltd., \\ Hacchonawate, Takatsuki, Osaka 569, Japan.
}

(Received September 24, 1980)

\begin{abstract}
An attempt was made to extend Ferry's and Renkin's theories establishing a very comprehensive and general theory that would explain the separation of particles in solution by ultrafiltration with a polymeric membrane. In addition to the pore size distribution $N(r)$ in the membrane ( $r$, pore radius), four factors were considered: (1) the sieving effect due to collision between particles in the filtrand with the pore wall or the pore edge of the membrane (steric factor), (2) viscous interaction between the particle and the pore wall (viscous factor), (3) the hydrodynamic effect on the particle (fractionation factor), and (4) intermolecular forces between the particle and the membrane (intermolecular factor). The ratio $\varphi(a)$ of the particle concentration in the filtrate to that of the filtrand ( $a$, radius of particle) was expressed as a function of particle size, with the filtration conditions including the flow rate (shear rate) of the filtrand, and the membrane characteristics $(N(r)$ and porosity). The validity of the proposed theory was confirmed by the experiment.
\end{abstract}

KEY WORDS Permselectivity / Porous Polymeric Membrane /
Ultrafiltration / Pore Size Distribution / Straight-Through Pore / Artificial
Kidney /

For the past decade, attention has been increasingly focussed on the science and technology of separating particles dispersed or dissolved in liquids by means of a porous polymeric membrane. It is considered that separation of particles by size using a membrane is governed by the following two mechanisms: (a) the material (particle) passes through a kind of void formed temporarily by the thermal molecular motion of the polymer constituting the membrane. This is called the "dissolutiondiffusion mechanism," which is predominant when pores are not observable by an electron microscope; (b) the particles with a radius $a$ smaller than the radius $r$ of the pores in the membrane can pass only through permanent pores and are separated according to the molecular size. This is often called the "molecular sieving mechanism." It is recognized that, in general, as the pore radius $r$ decreases, the intermolecular force between the polymeric material constituting the membrane and the solute molecules in the filtrand plays a very important role in the filtration governed by the dissolution-diffusion

mechanism. ${ }^{2-4}$

In the ultrafiltration process, the mechanism (b) is expected to predominate. However, Ferry ${ }^{5}$ verified theoretically that particles smaller in size than the pore cannot always pass through the membrane. This indicates that the molecular sieving mechanism is not completely adequate (see eq 5). Renkin ${ }^{6}$ modified Ferry's theory by taking into consideration the viscous force acting on particles moving in the pores (see eq 6). According to these theories, the ratio of the particle concentration of the filtrate to that of filtrand, $\varphi(a)$ ( $a$ is radius of the particle), depends only on the ratio $k$ of the radius $a$ of the particle to that of the pore $r$. The point is that these theories neglected, (a) the effect of the flow of the filtrand (the flow rate or shear rate) on $\varphi(a)$, (b) the intermolecular forces between particle-membrane and particle-solvent and (c) the pore size distribution $N(r)$ of the membrane. Unfortunately, these three factors are not negligible from the experimental point of view. For example, $\varphi(a)$ depends markedly on the flow rate (see Figure 21) and even if 
solutes with similar molecular weights (i.e., approximately similar $a$ values) are filtered, $\varphi(a)$ differs from solute to solute (see Figure 7 of ref 7). Also, the actual membranes have wide pore size distribution (see Figure 14).

In order to critically evaluate the reliability of the theories proposed so far, we should first determine the pore radius $r$ and the particle radius $a$ accurately. The mean pore size should also be determined. But owing to the absence of a method for the quantitative evaluation of pore characteristics, no study which provides this information has been possible except for our recent systematic approach. ${ }^{8}$

In the course of a previous study on the filtrationtype artificial kidney for hemoperfusion, in which the porous membrane having a mean pore size $\left(2 \bar{r}_{4}\right.$ defined below) of $90 \mathrm{~nm}$ was utilized, Kamide et al. ${ }^{9}$ observed a great difference in the composition of the perpendicular flow filtration with that of the parallel flow filtration. These are schematically represented in Figure 1. In the former (Figure 1a) the blood is almost stationary, but in the latter (Figure 1b), the blood moves continuously parallel to the membrane surface.

The previous theories by Ferry ${ }^{5}$ and Renkin ${ }^{6}$ fail to explain this difference. Kamide et al. ${ }^{8}$ explain that in the parallel flow filtration process, fractionation occurs on the basis of a particle size; the larger particles have a tendency to accumulate to the position of the maximum flow rate (i.e., the center of the flow in the case of cylindrical laminar flow), resulting in a compositional local difference in the filtrand.

The experimental results on the filtration-type artificial kidney suggest that in deriving the fundamental equation for $\varphi(a)$, the following five variables should be taken into account: (1) radius of solute particle, (2) mean pore size and pore-size distribution of the membrane, (3) the flow rate of the filtrand or its shear rate, (4) the pressure applied to the membrane surface, and (5) temperature.

In this paper, we attempt to develop a general theory of permselectivity by taking into account the pore size distribution in the membrane, and to compare it with experimental results.

\section{THEORETICAL BACKGROUND}

Figure 2 illustrates that the concentration of particles of radius $a$ changes with distance from the

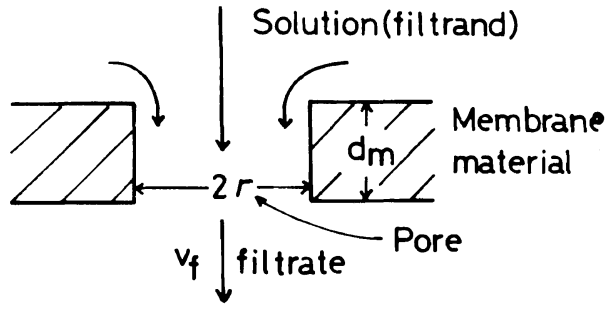

(a) Perpendicular filtration

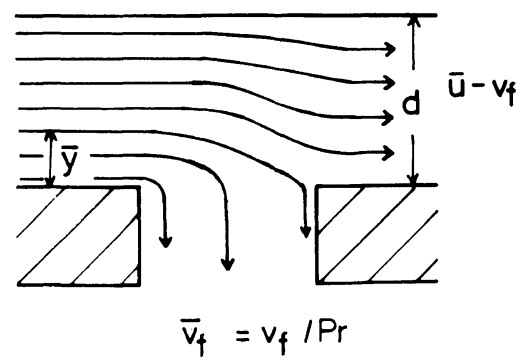

(b) Pararell filtration

Figure 1. Schematic representation of perpendicular and parallel flow filtrations: (a) perpendicular flow filtration; (b) parallel flow filtration. Arrows indicate the vector of flow velocity. The shaded part represents the membrane of thickness $d_{\mathrm{m}}$; $d$, thickness of the slit of flow; $r$, pore radius; $v_{\mathrm{f}}$, filtration rate per unit area of membrane; $P_{\mathrm{r}}$, membrane porosity; $\bar{v}_{\mathrm{f}}$, mean flow rate of filtrate in a pore; $\bar{u}$, mean flow rate of solution (filtrand).

membrane surface. In this figure, 0 indicates the position of the membrane surface, $d_{\mathrm{m}}$, the thickness of the membrane, $d$, the width of the filtrand flow, $\bar{C}_{\mathrm{a}}$, the mean particle concentration of the filtrand not generally equal to the concentration of the filtrand when at a distance of $1 / 2 d$ from the membrane surface, and $\bar{C}_{\mathrm{as}}$, the mean concentration of particles in the filtrand that may pass through a pore. The change in the filtrand concentration from $\bar{C}_{\mathrm{a}}$ to $\bar{C}_{\mathrm{as}}$ as shown in Figure 2 may originate not only from fractionation (referred to as the fractionation factor) occuring on the basis of particle size, but also from molecular interaction (intermolecular factor). The latter factor can be represented by three kinds of interactions, i.e., polymer molecule constituting the membrane-particle, particle-solvent, solvent-polymer molecule. $C_{\mathrm{a} i}$ is the particle concentration at the entrance of a pore. The concentration change from $\bar{C}_{\text {as }}$ to $C_{\text {ai }}$ results from the difference in the effective pore area of the solvent and particle passing through the membrane (referred to 


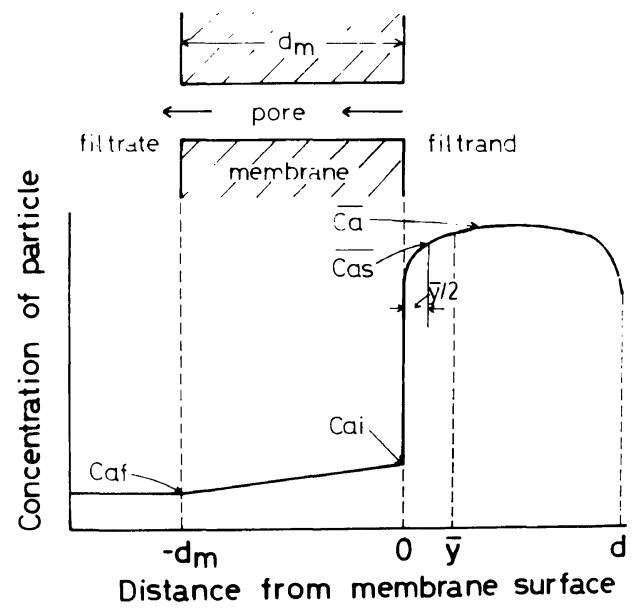

Figure 2. Schematic representation of the concentrational distribution of particles of having radius $a$ as a function of the distance from the membrane surface: $d_{\mathrm{m}}$, thickness of membrane; $d$, width of gap of filtrand flow; 0 , position of membrane surface; $\bar{C}_{\mathrm{a}}$, mean particle concentration in filtrand; $\bar{C}_{\text {as }}$, mean surface concentration of particles in the filtrand that can pass through the pore; $C_{\mathrm{ai}}$, particle concentration at the entrance of the pore; $C_{\text {af }}$, particle concentration in the filtrate.

as the steric factor). $C_{\mathrm{af}}$ is the particle concentration in the filtrate. When the solvent molecule and the particle pass through a pore, the difference in velocity between these gives rise to the change in the concentration from $C_{\mathrm{a} i}$ to $C_{\mathrm{af}}$. This velocity difference is due to the viscosity of the fluid in the pore. The change from $C_{\mathrm{ai}}$ to $C_{\mathrm{af}}$ is referred to as the viscous factor.

If the effective pore areas of the solvent and the particle are represented by $S_{\mathrm{v}}$ and $S_{\mathrm{a}}$, respectively, then $C_{\mathrm{ai}}$ is given by

$$
C_{\mathrm{ai}} /\left(1-C_{\mathrm{ai}}\right)=S_{\mathrm{a}} \bar{C}_{\mathrm{as}} /\left[S_{\mathrm{v}}\left(1-\bar{C}_{\mathrm{as}}\right)\right]
$$

Since both $C_{\mathrm{ai}}$ and $\bar{C}_{\mathrm{as}}$ are usually less than 0.01 , eq 1 may be rewritten as

$$
C_{\mathrm{ai}} / \bar{C}_{\mathrm{as}}=S_{\mathrm{a}} / S_{\mathrm{v}}
$$

We define $\varphi(a)$ as the ratio of the concentration $C_{\text {af }}$ of particles with radius $a$ in the filtrate to that in the filtrand before filtration, $\bar{C}_{\mathrm{a}}$ (i.e., the feed concentration),

$$
\varphi(a)=C_{\mathrm{af}} / \bar{C}_{\mathrm{a}}
$$

$\varphi(a)$ is a directly measurable quantity. When the osmotic pressure on the membrane $\Delta \pi$ is zero, the concentration ratio $\varphi(a)$ is related to the reflection coefficient $\sigma(a)$ by

$$
\varphi(a)=1-\sigma(a)
$$

The condition $\Delta \pi=0$ is experimentally satisfied and assumed throughout this paper. Here, both the particles dispersed and the molecules dissolved in the solution are referred to as "particles."

Ferry ${ }^{5}$ was the first to propose a theory for interpreting the permselectivity of the ultrafiltration process. For a process in which a solution containing particles of radius $a$ is passed through a membrane having a pore radius of $r$, Ferry assumed that (a) the pore is perfectly cylindrical and perpendicular to the membrane surface, (b) there is no clogging or adsorption of particles on the membrane surface, (c) the direction of the filtrand flow is perpendicular to the membrane surface and only the particles, whose centers of gravity are within a distance of $r-a$ from the center of the pore, can pass through the membrane (this is the so-called steric hindrance effect or molecular sieving effect), and (d) the flow of the liquid inside the pore is a onedimentional Poiseuille flow. The relationship he obtained between $\varphi(a)$ and $a / r$ for this membrane with a pore radius of $r$ is given by

$$
\varphi(a)=2\{1-(a / r)\}^{2}-\{1-(a / r)\}^{4}
$$

The velocity of the particles in the pore is retarded by a viscous force less than that of the solvent. ${ }^{11}$ Renkin $^{6}$ modified eq 5 by taking into consideration this effect (i.e., the viscous factor), so that,

$$
\begin{aligned}
\varphi(a)=\{[ & \left.1-(a / r)]^{2}-[1-(a / r)]^{4}\right\}[1-2.104(a / r) \\
+ & \left.2.09(a / r)^{3}-0.95(a / r)^{5}\right]
\end{aligned}
$$

According to eq 5 and 6 , where the nonuniformity of pore size is not considered, $\varphi(a)$ is expected to be a single function of the ratio $a / r$. This prediction is at variance with our experimental results on hemo-ultrafiltration, ${ }^{7,9}$ in which $\varphi(a)$ is a function not only of the ratio $a / r$ but also of the flow rate of the filtrand and pressure applied to the membrane. As a result, we undertook the present study.

We denote the number of pores having radii between $r$ and $r+\mathrm{d} r$ in a unit area of a membrane by $N(r) \mathrm{d} r$. Thus $N(r)$ is the frequency distribution of pore radius $r$. If the pore density $N$ is defined as the 
total number of pores in a unit area, $N$ may be represented by

$$
N=\int_{0}^{\infty} N(r) \mathrm{d} r
$$

The $i$ th mean pore radius $\bar{r}_{i}$ is defined by equation, ${ }^{8}$

$$
\overline{r_{i}}=\int_{0}^{\infty} r^{i} N(r) \mathrm{d} r / \int_{0}^{\infty} r^{i-1} N(r) \mathrm{d} r
$$

The porosity $P_{\mathrm{r}}$ is the volume fraction of pores in a membrane and, for a membrane consisting of straight-through cylindrical pores ${ }^{8}$ is given by

$$
P_{\mathrm{r}}=\pi \int_{0}^{\infty} r^{2} N(r) \mathrm{d} r=\pi \bar{r}_{2} \cdot \bar{r}_{1} \cdot N
$$

We define $\varphi(a, \delta)$ as $\varphi(a)$ in the case when $N(r)$ is represented by a $\delta$ function (in other words, the pore size distribution in the membrane is absolutely uniform). Assuming that the flow in the pores obeys Poiseuille law and that the overall flux from the membrane is given by the sum of the individual fluxes, we obtain

$$
\varphi(a)=\int_{0}^{\infty} \varphi(a, \delta) r^{4} N(r) \mathrm{d} r / \int_{0}^{\infty} r^{4} N(r) \mathrm{d} r
$$

When the values of $N(r)$ and $\varphi(a, \delta)$ are known in advance, $\varphi(a)$ can be calculated by using eq 10 .

For a membrane having a uniform pore size distribution, $\varphi(a)$ in eq 3 reduces to $\varphi(a, \delta)$, and can be rewritten as

$$
\varphi(a, \delta)=C_{\mathrm{af}} / \bar{C}_{\mathrm{a}}=\left(C_{\mathrm{af}} / C_{\mathrm{ai}}\right)\left(C_{\mathrm{ai}} / \bar{C}_{\mathrm{as}}\right)\left(\bar{C}_{\mathrm{as}} / \bar{C}_{\mathrm{a}}\right)
$$

The definitions of $C_{\mathrm{a}}, C_{\mathrm{ai}}, \bar{C}_{\mathrm{as}}$, and $\bar{C}_{\mathrm{a}}$ are illustrated in Figure 2. It should be noted that $C_{\mathrm{af}}$ and $C_{\mathrm{a}}$ are the only observable quantities. For convenience, the concentration ratios $\left(C_{\mathrm{af}} / C_{\mathrm{ai}}\right),\left(C_{\mathrm{ai}} / \bar{C}_{\mathrm{as}}\right)$ and $\left(\bar{C}_{\mathrm{as}} / \bar{C}_{\mathrm{a}}\right)$ are expressed as $\varphi_{\mathrm{v}}(a, \delta), \varphi_{\mathrm{s}}(a, \delta)$ and $\varphi_{\mathrm{fm}}(a, \delta)$, respectively,

$$
\left.\begin{array}{l}
\varphi_{\mathrm{v}}(a, \delta)=C_{\mathrm{af}} / C_{\mathrm{ai}} \\
\varphi_{\mathrm{s}}(a, \delta)=C_{\mathrm{a}} / \bar{C}_{\mathrm{as}} \\
\varphi_{\mathrm{fm}}(a, \delta)=\bar{C}_{\mathrm{as}} / \bar{C}_{\mathrm{a}}
\end{array}\right\}
$$

Equation 11 is simplified to eq 13, using the above parameters

$$
\varphi(a, \delta)=\varphi_{\mathrm{s}}(a, \delta) \cdot \varphi_{\mathrm{v}}(a, \delta) \cdot \varphi_{\mathrm{fm}}(a, \delta)
$$

In eq $13, \varphi_{\mathrm{s}}(a, \delta), \varphi_{\mathrm{v}}(a, \delta)$, and $\varphi_{\mathrm{fm}}(a, \delta)$ denote the contribution of the steric, viscous, fractionation and intermolecular factors toward $\varphi(a, \delta)$, respectively.

By substituting eq 13 into eq 10 , we obtain

$$
\begin{aligned}
\varphi(a)= & \int_{0}^{\infty} \varphi_{\mathrm{s}}(a, \delta) \cdot \varphi_{\mathrm{v}}(a, \delta) \\
& \cdot \varphi_{\mathrm{fm}}(a, \delta) r^{4} N(r) \mathrm{d} r / \int_{0}^{\infty} r^{4} N(r) \mathrm{d} r
\end{aligned}
$$

Equation 14 is the fundamental equation for calculating $\varphi(a)$.

$\varphi_{\mathrm{s}}(a), \varphi_{\mathrm{v}}(a)$, and $\varphi_{\mathrm{fm}}(a)$ are, as in the case of eq 10 , defined by

$$
\begin{aligned}
\varphi_{\mathrm{s}}(a) & =\int_{0}^{\infty} \varphi_{\mathrm{s}}(a, \delta) r^{4} N(r) \mathrm{d} r / \int_{0}^{\infty} r^{4} N(r) \mathrm{d} r \\
\varphi_{\mathrm{v}}(a) & =\int_{0}^{\infty} \varphi_{\mathrm{v}}(a, \delta) r^{4} N(r) \mathrm{d} r / \int_{0}^{\infty} r^{4} N(r) \mathrm{d} r \\
\varphi_{\mathrm{fm}}(a) & =\int_{0}^{\infty} \varphi_{\mathrm{fm}}(a, \delta) r^{4} N(r) \mathrm{d} r / \int_{0}^{\infty} r^{4} N(r) \mathrm{d} r
\end{aligned}
$$

and also the following two parameters $\overline{\varphi_{\mathrm{s}}(a) \cdot \varphi_{\mathrm{v}}(a)}$, and $\overline{\bar{\varphi}}_{\mathrm{fm}}(a)$ can be defined by,

$$
\begin{aligned}
\overline{\varphi_{\mathrm{s}}(a) \cdot \varphi_{\mathrm{v}}(a)}= & \int_{0}^{\infty} \varphi_{\mathrm{s}}(a, \delta) \\
& \cdot \varphi_{\mathrm{v}}(a, \delta) r^{4} N(r) \mathrm{d} r / \int_{0}^{\infty} r^{4} N(r) \mathrm{d} r
\end{aligned}
$$

and,

$$
\overline{\bar{\varphi}}_{\mathrm{fm}}(a)=\varphi(a) / \overline{\varphi_{\mathrm{s}}(a) \cdot \varphi_{\mathrm{v}}(a)}
$$

Here it should be noted that the product of $\varphi_{\mathrm{s}}(a)$, $\varphi_{\mathrm{v}}(a)$, and $\varphi_{\mathrm{fm}}(a)$ is generally not equal to $\varphi(a)$.

When $\varphi_{\mathrm{fm}}(a, \delta)$ is nearly equal to unity, $\varphi(a)$ can be approximated by $\overline{\varphi_{\mathrm{s}}(a) \cdot \varphi_{\mathrm{v}}(a)}$ and when both $\varphi_{\mathrm{fm}}(a, \delta)$ and $\varphi_{\mathrm{f}}(a, \delta)$ are nearly equal to unity, $\varphi(a)$ can be approximated by $\varphi_{\mathrm{s}}(a)$. Consequently, if we derive the theoretical equations for $\varphi_{\mathrm{s}}(a, \delta)$, $\varphi_{\mathrm{v}}(a, \delta)$, and $\varphi_{\mathrm{fm}}(a, \delta)$, the $\varphi(a)$ for a membrane of a known $N(r)$ can be calculated by eq 14. In the following section, we will derive these three concentration ratios.

\section{Steric Factor $\varphi_{\mathrm{s}}(a, \delta)$}

A typical parallel flow pattern of the filtrand flow on the membrane has already been demonstrated in Figure $1 \mathrm{~b}$. The overall filtration rate per unit area of the membrane surface is expressed by $v_{\mathrm{f}}$ and the mean velocity of the filtrate flowing in a pore $\bar{v}_{\mathrm{f}}$ coincides with the ratio of $v_{\mathrm{f}}$ to the porosity $P_{\mathrm{r}}$ of the membrane, i.e., $\bar{v}_{\mathrm{f}}=v_{\mathrm{f}} / P_{\mathrm{r}}$. Here, the collision 
angle $\alpha$, defined as the angle between the normal to the membrane surface and the direction of the flow of a particle, is given by (see Appendix I)

$$
\alpha=\tan ^{-1}\left\{\left(3 r \bar{u} P_{\mathrm{r}} / 2 v_{\mathrm{f}} \cdot d\right)^{1 / 2} \times 0.575 \pi\right\}
$$

where, $r$ is the pore radius, $\bar{u}$ is the average flow velocity of the filtrand and $d$ is the thickness of the flow (i.e., the gap width on the membrane). Ferry ${ }^{5}$ assumed $\alpha=0$. Equation 20 was derived on the assumption that (a) the pore is circular and transverse throughout the membrane, (b) the flow of the solution on the membrane surface (filtrand) is Poiseuillian and has a thickness $d$, (c) the component of the particle flow velocity parallel to the membrane surface equals that of the filtrand, where the so-called viscous effect on a particle may be ignored, since $a \ll d$, (d) $\tan \alpha$ is given by $\bar{u}_{\mathrm{p}} / \bar{v}_{\mathrm{f}}$, where $\bar{u}_{\mathrm{p}}$ is the average rate of the filtrand flow through a pore, (e) the radius of the solvent molecule may be ignored, in consideration of that of the particles, and (f) collision between particles and the membrane surface (or the pore wall) is perfectly elastic.

In Figure 3, the collision of particles in the filtrand against the membrane at an angle $\alpha$ is schematically represented. The broken line is the trace of the center of gravity of the particle at the moment of collision. Since it is obvious that when particles collide with region I of the membrane, the particles are not in the pore, and when particles collide with the pore wall defined as region III, the particles are in the pore. When the collision occurs in region II, the particles are either inside or outside the pore. If we can express the position of a particle in a pore following collision in terms of $a, r$, and $\alpha$, the effective pore area over which the particle passes through $S_{\mathrm{a}}$ can be calculated in the way shown below. For simplicity, we assume that the solvent constitutes the fluid body (i.e., continuous body) $(\alpha=0)$ and the radius of the solvent molecule in the solution is zero, i.e., $S_{v}=\pi r^{2}$.

Figure 4a shows a schematic representation of a situation in which a particle in the filtrand collides with the edge of the pore wall and is oriented in a unidirectional flow. The shaded parts in the figure indicate the membrane, 0 is the center of gravity of the particle at the moment of collision. Particles contact the edge of the pore indicated by $C . \overline{\mathrm{OA}}$ is the velocity vector of a particle before the collision, $\overline{\mathrm{OB}}$ is that following collision, and $\mathrm{F}$ is the point on the membrane surface at a distance $a$ from the edge

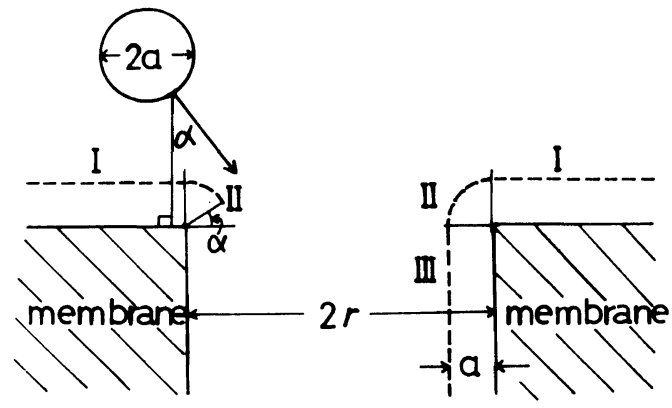

Figure 3. Schematic representation of the collision in a solution of a particle, of size is $2 a$, with the pore wall or the pore edge of a membrane: $a$,particle radius; $r$, pore radius; $\alpha$, collision angle, that is, the angle between the normal to the membrane surface and the flow direction of a particle; broken line, locus of the gravity center of the particle at the moment of collision with the membrane. Regions I, II, and III indicate positions where collision occurs with the membrane surface, the pore edge, and the pore wall, respectively.

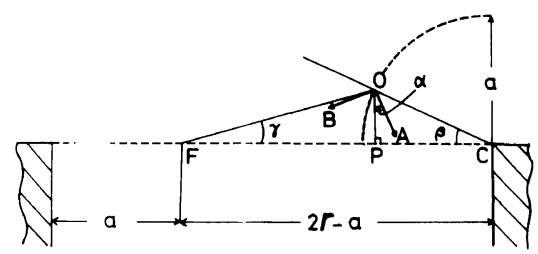

(a)

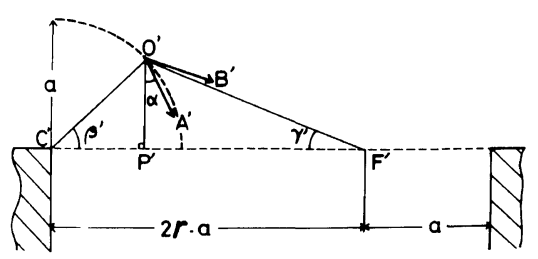

(b)

Figure 4. Schematic representation of the collision of a particle in the filtrand with the edge of the pore wall. (a) collision at the right hand side edge; the edge of the pore wall facing an anti-flow direction. (b) Collision at the left hand side edge; the edge faces to the flow direction. Shaded part, membrane; 0 and $0^{\prime}$, center of gravity of the particle at the moment of collision; $\overrightarrow{\mathrm{AC}}$ (in Figure 4(a)) and $C^{\prime} A^{\prime}$ (in Figure 4(b)), velocity vectors of the particle before collision. Other symbols are defined in the text.

of the pore. Line OP is drawn so as to intersect with line CF perpendicularly. $\beta$ is the angle $\angle$ OCF. $\gamma$ is 
the angle $\angle O F C$. Here, the positive values of the angles are measured clockwise and are limited to a range between $\pi$ and $-\pi$.

The relations $\overline{\mathrm{AO}}=\overline{\mathrm{BO}}$ can be derived from assumption ( $f$ ). If the center of gravity of a particle is below point $\mathrm{F}$ after colliding with the wall or the edge of a pore (the wall or the edge on the left-hand side in Figure 4a), the particle can pass through the pore. When the direction of $\overrightarrow{\mathrm{OB}}$ coincides with the direction of $\overrightarrow{\mathrm{OF}}$, angle $\beta$ is denoted by $\beta_{\mathrm{r}}$. Then $\beta_{\mathrm{r}}$ is given by

$$
\beta_{\mathrm{r}}=(\pi / 2-\alpha-\gamma) / 2
$$

where

$$
\gamma=\tan ^{-1}\left[a \cdot \sin \beta_{\mathrm{r}} /\left\{2 r-a\left(1+\cos \beta_{\mathrm{r}}\right)\right\}\right]
$$

Combination of eq 21 with eq 22 leads to,

$$
\beta_{\mathrm{r}}=\left[\pi / 2-\alpha-\tan ^{-1}\left[k \cdot \sin \beta_{\mathrm{r}} /\left\{2-k\left(1+\cos \beta_{\mathrm{r}}\right)\right\}\right]\right] / 2
$$

where

$$
k=a / r
$$

When $\beta>\beta_{\mathrm{r}}$, a particle following a collision can not pass through the pore, i.e., a particle, whose center of gravity is $\left(a \cdot \cos \beta_{\mathrm{r}}-a \cdot \sin \beta_{\mathrm{r}} \cdot \tan \alpha\right)\left(=D_{1}\right)$ from the edge of the pore (point $C$ in Figure 4(a)), can not pass through the pore.

In Figure $4 \mathrm{~b}$ is shown a situation in which a particle, parallel to the flow direction, collides with the edge of the pore wall. The prime mark is attached to $\mathrm{C}, \mathrm{F}, \mathrm{O}$ and $\mathrm{P}$ in order to distinguish particle collision with the left hand wall from that with the right hand wall. These primed letters have the same meaning as $\mathrm{C}, \mathrm{F}, \mathrm{O}$, and $\mathrm{P}$ in Figure 4a. Also, $\beta^{\prime}$ and $\gamma^{\prime}$ have the same meaning as $\beta$ and $\gamma$, respectively. The velocity vector of the particle before the collision is represented by $\mathrm{O}^{\prime} \mathrm{A}^{\prime}$ and that after the collision by $\mathrm{O}^{\prime} \mathrm{B}^{\prime}$. it is assumed that when a particle after the first collision collides with another pore wall, (in this case right-hand side) and with its center of gravity beneath point $\mathrm{F}^{\prime}$ can come into the pore. Thus, when $\alpha$ satisfies the condition $\alpha \geqq \gamma+2 \beta^{\prime}-\pi / 2$, the particle can pass through the pore. This condition can be rewritten in the form:

$$
\beta^{\prime} \leqq\left(\pi / 2-\gamma^{\prime}+\alpha\right) / 2
$$

A corresponding equation for $\gamma^{\prime}$ can be derived in exactly the same way as employed in the derivation of eq 22 and is given by

$$
\gamma^{\prime}=\tan ^{-1}\left\{k \cdot \sin \beta^{\prime} /\left[2-k\left(1+\cos \beta^{\prime}\right)\right]\right\}
$$

where, $k$ is defined by eq 24 .

The maximum value of $\beta^{\prime}$, i.e., $\beta_{1}{ }^{\prime}$, which satisfies eq 25 and 26 simultaneously is given by

$$
\begin{aligned}
\beta_{1}{ }^{\prime}=[\pi / 2 & +\alpha-\tan ^{-1}\{k \\
\cdot & \left.\left.\sin \beta_{1}{ }^{\prime} /\left[2-k\left(1+\cos \beta_{1}{ }^{\prime}\right)\right]\right\}\right] / 2
\end{aligned}
$$

Here, $\beta_{1}{ }^{\prime}$ should satisfy the relation; $\beta_{1}{ }^{\prime} \geqq \alpha$, which can be readily derived from the definition of $\beta_{1}{ }^{\prime}$.

Due to the collision with the pore edge, a particle, with its center of gravity within a distance of $\left(a \cdot \cos \beta_{1}{ }^{\prime}+a \cdot \sin \beta_{1}{ }^{\prime} \cdot \tan \alpha\right) \quad\left(=D_{2}\right)$ from the pore edge ( $C^{\prime}$ in Figure $\left.4 b\right)$ cannot pass through the pore.

Therefore, the effective pore area $S_{\mathrm{a}}$ is given by

$$
S_{\mathrm{a}}=\int_{-\sqrt{r^{2}-a^{2} \cos ^{2} \bar{\beta}}}^{\sqrt{r^{2}-a^{2} \cos ^{2} \beta}}(2 x-\bar{D}) \mathrm{d} z
$$

where

$$
\begin{gathered}
x^{2}+z^{2}=r^{2} \\
\bar{D}=D_{1}+D_{2}=2 a \cdot \cos \bar{\beta} \\
\cos \bar{\beta}=\cos \left(\frac{\beta_{1}{ }^{\prime}+\beta_{\mathrm{r}}}{2}\right) \\
\times\left\{\cos \left(\frac{\beta_{1}{ }^{\prime}-\beta_{\mathrm{r}}}{2}\right)+\sin \left(\frac{\beta_{1}{ }^{\prime}-\beta_{\mathrm{r}}}{2}\right) \tan \alpha\right\}
\end{gathered}
$$

Here, $x$ and $z$ represent the coordinates of the pore edge on the $X$ and $Z$ axes which are set up on the membrane surface, with the origin at the center of the circular pore. The upper and lower limits of the integration in eq 28 are the larger and smaller roots of the equation, $z^{2}=r^{2}-\bar{D}^{2} / 4$.

In Figure 5, the effective pore area (shaded area) calculated by the present theory, is compared with that determined by Ferry's theory, in which $\alpha=0$ and $\gamma=-\pi / 2$ are assumed. The latter assumption is obviously unrealistic. The Ferry's effective pore area is independent of $\bar{u}$, and smaller than the present one under $\bar{u}=0$. On the other hand, the area given by the present theory decreases with increasing $\bar{u}$. The solvent molecule constitutes a continuous body (fluid) (hence, $\alpha=0$ ) and its radius is so small that the effective area $S_{\mathrm{v}}$ for the solvent can be approximated by $\pi r^{2}$. Combination of eq 12, 2 and 28 , leads to 


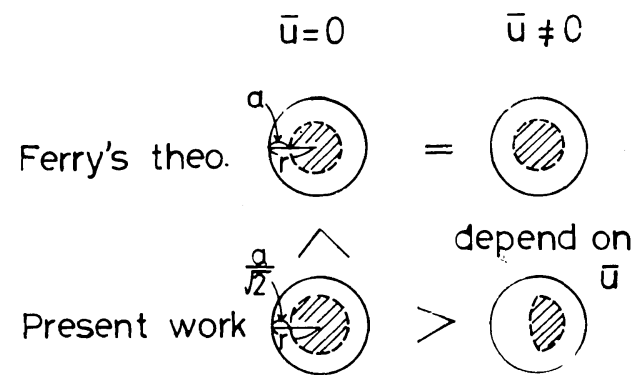

Figure 5. Comparison between Ferry's theory and the present theory in regard to the steric factor concerning to $\varphi(a)$ : (a), the Ferry's theory; (b), the present theory. The Four circles indicate pores, and the shaded area represents the effective pore area that allows particles to pass.

$$
\begin{aligned}
\varphi_{\mathrm{s}}(a, \delta)= & \int_{-\sqrt{r^{2}-a^{2} \cos ^{2} \bar{\beta}}}^{\sqrt{r^{2}-a^{2} \cos ^{2} \bar{\beta}}}(2 x-\bar{D}) \mathrm{d} z / \pi r^{2} \\
= & (2 / \pi)\left(\sin ^{-1} \sqrt{1-k^{2} \cos ^{2} \bar{\beta}}\right. \\
& \left.-k \sqrt{1-k^{2} \cos ^{2} \bar{\beta}} \cos \bar{\beta}\right)
\end{aligned}
$$

In Ferry's theory, the condition $\beta_{\mathrm{r}}=\beta_{1}{ }^{\prime}=0$ (accordingly, $\bar{\beta}=0$, see eq 31) was assumed regardless of $\alpha$ and $k$. Putting $\bar{\beta}=0$ into eq 32 results in the equation (see eq 11 of reference 5 ) for the steric factor obtained by Ferry 5. This is the case in which the flow is governed by diffusion.

\section{Viscous Factor $\varphi_{\mathrm{v}}(a, \delta)$}

The ratio $C_{\mathrm{af}} / C_{\mathrm{ai}}$ is equal ${ }^{1}$ to the ratio of the velocities of a particle $\left(v_{\mathrm{a}}\right)$ and a solvent molecule $\left(v_{\mathrm{s}}\right)$ in a pore, and hence $\varphi_{\mathrm{v}}(a, \delta)=v_{\mathrm{a}} / v_{\mathrm{s}}$. For a particle inside the pore, the net viscous force can be neglected. The ratio of $v_{\mathrm{a}} / v_{\mathrm{s}}$ under the above condition has already been given by Happel and Byren $^{12}$ and also given by Faxen ${ }^{11}$ in a more generalized form. Using the equation derived by Faxén, we obtain

$$
\begin{aligned}
\varphi_{\mathrm{v}}(a, \delta)= & v_{\mathrm{a}} / v_{\mathrm{s}} \\
= & 1-(2 / 3) k^{2}-0.1628 k^{5}-0.4059 k^{7} \\
& +0.5326 k^{9}+1.51 k^{10}+\cdots
\end{aligned}
$$

Faxén's equation holds for $k \leqq 0.6^{13}$ and hence, eq 33 is also applicable in this range of $k$. We must note that as pointed out previously by Bean ${ }^{1}$ Renkin ${ }^{6}$ applied Faxén's theory in order to explain the variation in $\varphi(a, \delta)$ caused by the viscous factor without a detailed understanding of the hydrodynamic situation. Thus, Renkin's theory (hence, eq 4) is not theoretically correct with regard to viscous factor.

Fractionation and Intermolecular Factors $\varphi_{\mathrm{fm}}(a, \delta)$

It has widely been believed without experimental evidence that the concentration of particles in the filtrand is constant irrespective of the distance from the membrane surface; that is, $\bar{C}_{\mathrm{a}}=\bar{C}_{\mathrm{as}}$ holds. When the filtrand flows in a thin gap, there appears a velocity gradient which gives rise to a concentration distribution in the direction normal to the membrane surface. In order to evaluate $\bar{C}_{\text {as }}$ the thickness $\bar{y}$ of the filtrand that can enter into a pore and the change in the particle concentration with the distance should be given in advance.

As is shown in Appendix I, $\bar{y}$ is given by

$$
\begin{gathered}
\bar{y}=\left(\frac{v_{\mathrm{f}} \cdot d \cdot r}{P_{\mathrm{r}} \cdot U_{\max }}\right)^{1 / 2} \times 0.87=\left(\frac{v_{\mathrm{f}} \cdot r}{P_{\mathrm{r}} \cdot \dot{\gamma}}\right)^{1 / 2} \times 1.739 \\
\text { when } U_{\max } \neq 0
\end{gathered}
$$

with

$$
U_{\max }=1.5 \bar{u} \quad \text { and } \quad \dot{\gamma}=6 \bar{u} / d
$$

where, $U_{\max }$ is the maximum rate of the Poiseuille flow of the filtrand and $\dot{\gamma}$ is the shear rate of filtrand on the membrane surface. When $U_{\max }=0$ (i.e., $\dot{\gamma}=0$ ), $\bar{y}$ should be regarded as a parameter only on the filtration time.

Figure 6 shows the shear rate dependence of $\bar{y}$ calculated by eq 34 for membranes with a constant porosity $\left(P_{\mathrm{r}}=0.75\right)$ but with various $r$ values under a constant overall filtration rate $v_{\mathrm{f}}=3.3 \times 10^{-4}(\mathrm{ml}$ $\mathrm{cm}^{-2} \mathrm{~s}^{-1}$ ). These conditions approximately correspond to actual cases. The combination of $r / P_{\mathrm{r}}=0.1 \mu \mathrm{m}$ and $\dot{\gamma}=100\left(\mathrm{~s}^{-1}\right)$ is a typical experimental condition, under which $\bar{y}$ is nearly equal to a pore radius in practical filtration experiments. When the intermolecular interaction $A_{\mathrm{a}, \mathrm{s}}$ is negligibly small, the change in concentration of the particles in the solution (filtrand) can be explained only by the fractionation factor. We refer to $\varphi_{\mathrm{fm}}(a, \delta)$ in this case as $\varphi_{\mathrm{f}}(a, \delta)$; i.e., it is defined by $\varphi_{\mathrm{f}}(a, \delta)=\bar{C}_{\mathrm{as}} / \bar{C}_{\mathrm{a}}=\varphi_{\mathrm{fm}}(a, \delta)$ when $A_{\mathrm{a}, \mathrm{s}}=0$

On the other hand, when the flow rate of the filtrand is negligibly small (that is, $\dot{\gamma} \fallingdotseq 0$ ), as in the case of 


\section{K. KAMIDE and S. MANABE}

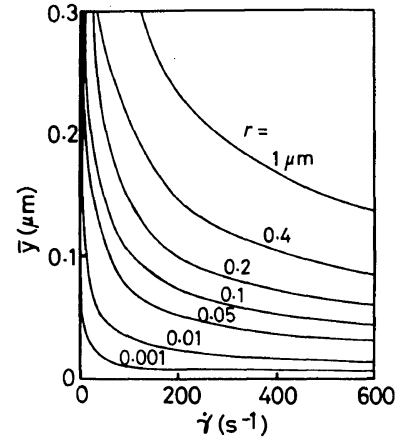

Figure 6. Shear rate dependence of $\bar{y}$. The numbers denote the values of pore radii in $\mu \mathrm{m}$. $\gamma$ is the shear rate of the filtrand on the membrane. $\bar{y}$ is thickness of the filtrand which can pass through a pore and is given by eq 34 .

perpendicular flow filtration, the concentration change in the filtrand above the membrane surface is caused by intermolecular interaction. Thus, we define $\varphi_{\mathrm{m}}(a, \delta)$ as follow,

$$
\varphi_{\mathrm{m}}(a, \delta)=\bar{C}_{\mathrm{as}} / \bar{C}_{\mathrm{a}} \quad \text { when } \quad \dot{\gamma}=0
$$

When both $\dot{\gamma}$ and the intermolecular interaction are not negligible, $\varphi_{\mathrm{fm}}(a, \delta)$ is assumed to be represented by the product, $\varphi_{\mathrm{f}}(a, \delta) \cdot \varphi_{\mathrm{m}}(a, \delta)$. Thus,

$$
\begin{aligned}
\varphi_{\mathrm{fm}}(a, \delta) & =\varphi_{\mathrm{f}}(a, \delta) \cdot \varphi_{\mathrm{m}}(a, \delta) \\
\text { when } \quad \dot{\gamma} & \neq 0, \quad \text { and } A_{\mathrm{a}, \mathrm{s}}
\end{aligned}
$$

Molecular fractionation in a capillary flow of polymer solution was observed for polyethylene. ${ }^{14}$ This type of fractionation must appear in a solution containing many particles varying in size. When a particle, at a given instant, is at a distance $y$ from $a$ the capillary wall, the velocity component $v(y)$ in the radial direction, $v(y)$, is derived by a modification of the experimental relation obtained by Goldsmith and Mason ${ }^{15}$ (see eq $7_{1}$ of ref 15), and yields,

$$
\begin{aligned}
v(y)= & B U_{\max }^{\prime}{ }^{2}\left(2 a / d^{\prime}\right)^{2.84} \\
& \times\left\{\left(d^{\prime}-2 y\right) / d^{\prime}\right\}\left\{1-(2 / d-y) / R\left(a, d^{\prime}\right)\right\}
\end{aligned}
$$

with

$$
R\left(a, d^{\prime}\right)=-0.75 a+0.77 d
$$

where $B$ is a constant independent of $y, a$, the velocity of fluid at the center of the cylinder $U_{\max }^{\prime}$, and the cylinder diameter $d^{\prime}$. The prime in eq 39 and 40 indicates the values for the capillary flow. We assume that eq 39 and 40 also hold for the two dimensional Poiseuille flow; that is, $d^{\prime}$ and $U_{\max }^{\prime}$ can be replaced by $d$ and $U_{\max }$, respectively. Then $v(y)$ increases with increasing $U_{\max }$. The larger the size of a particle $(a)$, the larger is the $v(y)$. In other words, a larger particle moves faster than the smaller one toward the center of the flow near the membrane surface. We denote the diffusion coefficient of a particle with radius $a$, by $D_{\mathrm{a}}$ and the concentration of a particle at $y$ from the membrane surface in the filtrand by $C(y)$. We then can derive from the mass conservation law,

$$
\frac{\partial C(y)}{\partial t}=-\frac{\partial C(y)}{\partial y} v(y)-\frac{\partial v(y)}{\partial y} C(y)+\frac{\partial^{2} C(y)}{\partial y^{2}} D_{\mathrm{a}}
$$

Equation 41 can be solved under a steady-state conditions. (see Appendix II). The solution is

$$
\begin{aligned}
C(y)= & \bar{C}_{\mathrm{a}} \exp \left\{-\left(B / 4 D_{\mathrm{a}}\right) \dot{\gamma}^{2}(2 a / d)^{2.84}\right. \\
& \left.\times(1-2 \bar{y} / d)^{2}[1-d(1-\bar{y} / d) / 3 R(a, d)]\right\}
\end{aligned}
$$

We approximate $\bar{C}_{\text {as }}$ in eq 12 by the value of $C(y)$ at $y=\bar{y} / 2$, that is, we put $\bar{C}_{\text {as }}=C(1 / 2 \bar{y})$. Substitution of the eq 42 into the definition equation of $\varphi_{\mathrm{f}}(a, \delta)$ given by eq 36 yìelds eq $43\left(A_{\mathrm{a}, \mathrm{s}}=0\right)$,

$$
\varphi_{\mathrm{f}}(a, \delta)=1-k_{\mathrm{f}} \dot{\gamma}^{2}(2 a / d)^{2.84}
$$

where

$$
k_{\mathrm{f}}=\left(B d / 4 D_{\mathrm{a}}\right)(1-\bar{y} / d)^{2}\{1-\mathrm{d}(1-\bar{y} / d) / 3 R(a, d)\}
$$

Inspection of eq 43 indicates that $\varphi_{\mathrm{f}}(a, \delta)$ decreases with increasing $\dot{\gamma}$ and also with increasing particle size $a$. The pore size influences $\varphi_{\mathrm{f}}(a, \delta)$ indirectly through $\bar{y}$ as can be seen from eq 44 . The pore size dependence of $\varphi_{\mathrm{f}}(a, \delta)$ is negligibly small for $\bar{y}>10 \mathrm{~nm}$, therefore, we find that the particle concentration in the filtrand changes significantly with the fractionation factor for all porous membranes regardless of pore size. Here, the molecular interaction energy between the particle in the filtrand and the molecule constituting the membrane is referred to as $W_{\mathrm{a}, \mathrm{p}}$ and that between the solvent molecule and the particle as $W_{\mathrm{sp}}$. The energy difference $\Delta W_{\mathrm{a}, \mathrm{s}}\left(=W_{\mathrm{a}, \mathrm{p}}-W_{\mathrm{s}, \mathrm{p}}\right)$ between these two interaction energies may give rise to the change in the solute concentration in the vicinity of the membrane surface. In a non-electrolyte solute solution, this energy difference $\Delta W_{\mathrm{a}, \mathrm{s}}$ may mainly arise from van der Waals interactions. Thus, $\Delta W_{\mathrm{a}, \mathrm{s}}$ can be es- 
timated by using the coupling constants in the London force $A_{\mathrm{a}, \mathrm{p}}$ and $A_{\mathrm{s}, \mathrm{p}} . W_{\mathrm{a}, \mathrm{p}}$ and $W_{\mathrm{s}, \mathrm{p}}$ are given by eq 45 and 46 under the condition $y \gg a,{ }^{16}$

$$
W_{\mathrm{a}, \mathrm{p}} \fallingdotseq-\left(A_{\mathrm{a}, \mathrm{p}} / 6\right) a /(y-a)
$$

and

$$
W_{\mathrm{s}, \mathrm{p}} \fallingdotseq-\left(A_{\mathrm{s}, \mathrm{p}}\right) r_{\mathrm{s}} /\left(y-r_{\mathrm{s}}\right)
$$

where, $r_{\mathrm{s}}$ is the radius of the solvent molecule. From eq 45 and 46 , we obtain,

$$
\Delta W_{\mathrm{a}, \mathrm{s}}=-\left(A_{\mathrm{a}, \mathrm{s}} / 6\right)(\bar{a} / y)
$$

where

$$
A_{\mathrm{a}, \mathrm{s}} \cdot \bar{a} \equiv A_{\mathrm{a}, \mathrm{p}} \cdot a-a_{\mathrm{s}, \mathrm{p}} \cdot r_{\mathrm{s}}
$$

When $\dot{\gamma}=0$ and the steady state is realized, the concentration distribution of particles as a function of the distance from the membrane surface may be described by the Boltzmann distribution law,

$$
C(y)=\bar{C}_{\mathrm{a}} \exp \left[\left(A_{\mathrm{a}, \mathrm{s}} \cdot \bar{a}\right) /(6 k T \cdot y)\right]
$$

$\bar{C}_{\text {as }}$ is approximated by the concentration at $y=\bar{y} / 2$ (i.e., $C(\bar{y} / 2)$ ), and is given by,

$$
\bar{C}_{\mathrm{as}}=\bar{C}_{\mathrm{a}} \exp \left[\left(A_{\mathrm{a}, \mathrm{s}} \cdot \bar{a}\right) /(3 k T \cdot \bar{y})\right]
$$

On substituting eq 50 into eq 37 , we obtain

$$
\begin{aligned}
\varphi_{\mathrm{m}}(a, \delta) & =\exp \left[\left(A_{\mathrm{a}, \mathrm{s}} \cdot \bar{a}\right) /(3 k T \cdot \bar{y})\right] \\
& \simeq 1+\left[\left(A_{\mathrm{a}, \mathrm{s}} \cdot \bar{a}\right) /(3 k T \cdot \bar{y})\right]
\end{aligned}
$$

Substitution of eq 43 and 51 into eq 38 yields

$$
\varphi_{\mathrm{fm}}(a, \delta)=\left[1-k_{\mathrm{f}} \dot{\gamma}^{2}(2 a / d)^{2.84}\right]\left[1+A_{\mathrm{a}, \mathrm{s}} \bar{a} /(3 k T \cdot \bar{y})\right]
$$

Combination of the $\varphi_{\mathrm{s}}(a, \delta)$ (eq 32), $\varphi_{\mathrm{v}}(a, \delta)$ (eq 33), and $\varphi_{\mathrm{fm}}(a, \delta)$ (eq 52) leads

$$
\begin{aligned}
\varphi(a, \delta)= & (2 / \pi)\left(\sin ^{-1} \sqrt{1-k^{2} \cos ^{2} \bar{\beta}}\right. \\
- & \left.k \sqrt{1-k^{2} \cos ^{2} \bar{\beta}} \cdot \cos \bar{\beta}\right) \\
& \times\left[1-(2 / 3) k^{2}-0.4059 k^{7}+0.5326 k^{9}\right. \\
+ & \left.\left.1.51 k^{10}+\cdots\right)\right]\left[1-k_{\mathrm{f}} \dot{\gamma}^{2}(2 a / d)^{2.84}\right] \\
& \times\left[1+A_{\mathrm{a}, \mathrm{s}} \cdot \bar{a} /(3 k T \cdot \bar{y})\right]
\end{aligned}
$$

This is the most generalized equation that describes the permselectivity of particles in the ultrafiltration process.

\section{EXPERIMENTAL}

\section{Membrane}

In order to examine the applicability of the theory proposed above, three types of membrane were used. (a) Four commercially available polycarbonate porous membranes "Nuclepore" manufactured by General Electric Company (USA) (sample

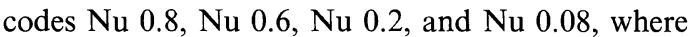
the number attatched to $\mathrm{Nu}$ stands for the mean pore size in $\mu \mathrm{m}$ informed by the producer) were used as the model sample having straight through cylindrical pore. (b) Three cellulose acetate membranes differing in pore size (sample codes SF 0.3, SF 0.2, and SF 0.09 , in this case $0.3,0.2,0.09$ indicate nominal value of mean pore size in $\mu \mathrm{m}$ ) were prepared in our laboratory as model samples with spherical pore. ${ }^{18}$ (c) The cellulose membrane for an artificial kidney manufactured by Enka AG (W. Germany) "Cuprophan" was used as the model sample with pore sizes less than $10 \mathrm{~nm}$.

\section{Characterization of Porous Membrane}

The frequency pore size distribution for all sample membranes, $N(r)$ except for Cuprophan were determined by the mercury intrusion method, and the scanning electron microscopic method. ${ }^{18}$ The $i$ th mean pore radius $\bar{r}_{\mathrm{i}}$ (see eq 8 ) was calculated by substituting the experimental $N(r)$ data into eq 8 . The porosity $P_{\mathrm{r}}$ of the membrane was estimated from the apparent density of the membrane. ${ }^{18}$ The characteristic values of the membranes are summarized in Table I.

\section{Numerical Calculation of Various Concentration Ratios}

Two analytical functions were chosen in order to represent the pore size distribution $N(r)$ of an actual membrane:

(a) $N(r)=N \delta(r-\bar{r})$;

where $\delta(x)$ is a $\delta$ function of variable $x$ (54)

(b) exponential function $N(r)=k_{\mathrm{b}} r^{\mathrm{b}}$

Here, $k_{\mathrm{b}}$ is a constant independent of $r$ and $\mathrm{b}$ is a constant integer ranging from -4 and 5. $\varphi_{\mathrm{s}}(a, \delta)$ and $\varphi_{\mathrm{v}}(a, \delta)$ were calculated by putting the given values of $k$ and $\alpha$ into eq 32 and 33, respectively.

Then, $\varphi_{\mathrm{s}}(a), \varphi_{\mathrm{v}}(a)$ and $\overline{\varphi_{\mathrm{s}}(a) \cdot \varphi_{\mathrm{v}}(a)}$ were calculated 
Table I. Characterization of various membranes

\begin{tabular}{ccccccc}
\hline Characteristic value & Nu 0.8 & Nu 0.6 & Nu 0.2 & Nu 0.08 & SF 0.3 & SF 0.2 \\
\hline $\bar{r}_{(\mu \mathrm{m})}$ & 0.309 & 0.235 & 0.118 & 0.0560 & 0.11 & 0.092 \\
$\bar{r}_{2}(\mu \mathrm{m})$ & 0.331 & 0.252 & 0.132 & 0.0599 & 0.13 & 0.095 \\
$\bar{r}_{3}(\mu \mathrm{m})$ & 0.317 & 0.268 & 0.156 & 0.0656 & 0.16 & 0.100 \\
$\bar{r}_{4}(\mu \mathrm{m})$ & 0.323 & 0.325 & 0.183 & 0.0713 & 0.21 & 0.120 \\
$P_{\mathrm{r}}(\%)$ & 15.3 & 14.0 & 10.3 & 7.2 & 75 & 75 \\
$N\left(\mathrm{no} . / \mathrm{cm}^{2}\right) \times 10^{-8}$ & 0.542 & 0.91 & 2.1 & 12.5 & 22 & 25 \\
Thickness $(\mu \mathrm{m})$ & 13.0 & 13.0 & 13.0 & 6.0 & 130 & 125 \\
\hline
\end{tabular}

by using eq 15,16 , and 18 from $\varphi_{\mathrm{s}}(a, \delta)$ and $\varphi_{\mathrm{v}}(a, \delta)$ thus obtained and the given $N(r)$. Since $\varphi_{\mathrm{fm}}(a, \delta)$ given by eq 52 is nearly independent of $r, \varphi_{\mathrm{fm}}(a)$ does not depend on $N(r)$. The value of $\varphi_{\mathrm{fm}}(a)$ calculated for $N(r)=N \delta(r-\bar{r})$ can then be applied to any case of $N(r)$.

\section{Solute and Solvent}

Urea (mol wt $=60.06$, reagent grade, manufactured by Kishida Chemical Co. Ltd., Japan), creatinine $(\mathrm{mol} \mathrm{wt}=113.12$, biochemical grade, manufactured by E. Merck AG, W. Germany), vitamine $\mathrm{B}_{12}(\mathrm{~mol} \mathrm{wt}=1371$, biochemical grade, manufactured by E. Merck AG, W. Germany), heparin (mol $\mathrm{wt}=c a .10^{4}$, guaranteed reagent grade, manufactured by Nakarai Chemicals Ltd., Japan), and dextrans $\left(M_{w}=1 \times 10^{4}, 6 \times 10^{4}, 2 \times 10^{5}\right.$, and $2 \times 10^{6}$, manufactured by Pharmacia Fine Chemicals AB, Sweden) were used as solutes without further purification. Distrilled water was used as the solvent. Concentrations of the dilute aqueous solutions utilized as the filtrands ranged from 0.005 to $0.1 \mathrm{wt} \%$ and were sufficiently low to allow the osmotic pressure $\Delta \pi$ to be neglected considering the applied pressure $\Delta P$.

Figure 7 shows the apparatus for carrying out the filtration. ${ }^{6}$ The pressure difference $\Delta P$ applied to on both sides of the membrane was kept constant within the range of $10-300 \mathrm{mmHg}$ through out each filtration against and the flow rate of solution $\bar{u}$ was also kept constant in the range from 0 $500 \mathrm{ml} \mathrm{min}^{-1}$.

\section{Measurement}

The particle concentration in fluid was measured by spectroscopy (Hitachi spectrophotometer type 100-50, manufactured by Hitachi Ltd., Japan) and by differential refractometry (ALC/GPC 201, manufactured by Waters Associate Inc., USA).

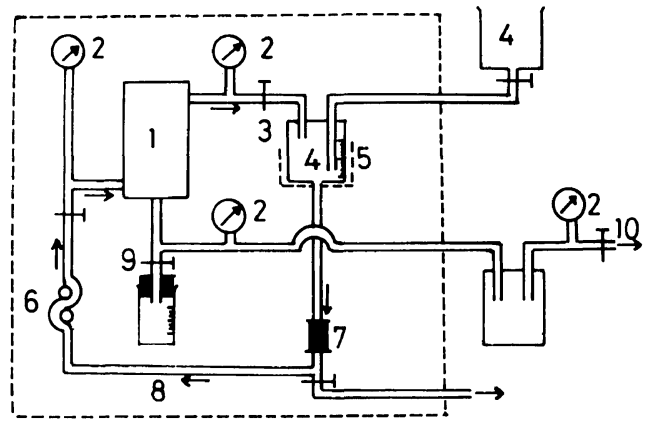

Figure 7. Apparatus for carrying out filtration ${ }^{9}$ : 1, filtration parts; 2 , pressure gauges; 3 , flow rate controlling bulb; 4 , storage tank for the solution; 5 , weighting balance; 6 , pump; 7 , prefilter; 8 , circulation line of solution (filtrand); 9 , recovery line of filtrate; 10 , vacuum line.

The dependence of the pressure loss (i.e., the pressure difference between the inlet and outlet of the filtration part 1 in Figure 7) on the flow rate indicated that the flow of the solution in the filtration part 1 was governed by the Poiseuille law, which is basic assumption in our theory.

\section{RESULTS AND DISCUSSION}

In Figure 8 the $\alpha$ dependence of $\beta_{\mathrm{r}}$ (full line) and $\beta_{1}{ }^{\prime}$ (broken line) for various $k$ values. $\beta_{\mathrm{r}}$ and $\beta_{1}{ }^{\prime}$ are accurately represented by

$$
\left.\begin{array}{c}
\beta_{\mathrm{r}}=\left(1-\pi k^{2} / 4\right)(\pi / 2-\alpha) / 2 \\
\beta_{1}{ }^{\prime}=\left(1-\pi k^{2} / 4\right)(\pi / 2+\alpha) / 2 \\
\text { for } \alpha \leqq\left\{\left(1-\pi k^{2} / 4\right) /\left(1+\pi k^{2} / 4\right)\right\}(\pi / 2) \\
\beta_{1}{ }^{\prime}=\alpha \\
\text { for } \alpha>\left\{\left(1-\pi k^{2} / 4\right) /\left(1+\pi k^{2} / 4\right)\right\}(\pi / 2)
\end{array}\right\}
$$

Equations 56 and $56^{\prime}$ allow the numerical calcu- 


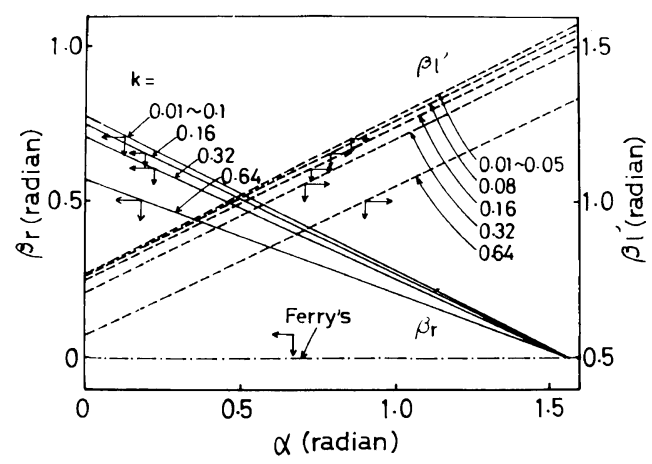

Figure 8. $\alpha$ dependences of $\beta_{\mathrm{r}}$ and $\beta_{1}$. The numbers denote $k$ value. The case of $\beta_{\mathrm{r}}=0$ shown by a dot-dash line corresponds to that derived by Ferry ${ }^{5}$.

lation of $\varphi_{\mathrm{s}}(a, \delta)$ (eq 32) to be simplified.

Theoretical Relationships between $\varphi(a)$ and $N(r)$, (Calculation)

$\delta$ Function Distribution. For simplicity we first consider eq 54 for $N(r)$. The combination of eq 10 , 54 , and 13 gives

$$
\varphi(a)=\varphi(a, \delta)=\varphi_{\mathrm{s}}(a, \delta) \cdot \varphi_{\mathrm{v}}(a, \delta) \cdot \varphi_{\mathrm{fm}}(a, \delta)(57)
$$

where $\varphi_{\mathrm{s}}(a, \delta), \varphi_{\mathrm{v}}(a, \delta)$, and $\varphi_{\mathrm{fm}}(a, \delta)$ are given by eq 32,34 , and 52 , respectively.

Figure 9(a) illustrates the particle size dependences of $\varphi_{\mathrm{s}}(a), \varphi_{\mathrm{v}}(a) \varphi_{\mathrm{fm}}(a, \delta), \overline{\varphi_{\mathrm{s}}(a) \cdot \varphi_{\mathrm{v}}(a)}$ and $\varphi(a)$ for the membrane with $\bar{r}=0.776 \mu \mathrm{m}$, calculated by taking $\alpha\left(=56^{\circ}\right), \quad \bar{r}(=0.776 \mu \mathrm{m}), \quad \dot{\gamma}(=1, \quad 10, \quad$ and $\left.100 \mathrm{~s}^{-1}\right), d(=3000 \mu \mathrm{m})$, and $k_{\mathrm{f}}\left(=1 \times 10^{7} / a \mathrm{~s}^{2}\right)$ and using the approximate equation, $\left(A_{\mathrm{a}, \mathrm{s}} / 3 k T\right)(\bar{a} / \bar{y})=$ $-(a / 3) . \bar{r}^{-1 / 2}$ obtained experimentally for aqueous solution of dextrans. Figure $9(\mathrm{~b})$ shows $\varphi_{\mathrm{s}}(a)$ (full line) and $\overline{\varphi_{\mathrm{s}}(a) \cdot \varphi_{\mathrm{v}}(a)}$ (broken line) $-\log a$ curves as a function of $\alpha$ for a membrane with $\bar{r}=0.776 \mu \mathrm{m}$. The numbers in Figure 9(a) indicate $\dot{\gamma}$ values in $s^{-1}$ and those in Figure 9(b) indicate $\alpha$ values in degrees. Inspection of Figure 9 leads to the following conclusions. (1) With an increase in $a$, all five concentration ratios in Figure 9(a) decrease monotonically. (2) $\varphi_{\mathrm{v}}(a)$ is larger than 0.9 for $k \leqq 0.4$, suggesting that the contribution of the viscous factor to $\varphi(a)$ is negligible in this range of $k$. (3) The dependence of $\varphi_{\mathrm{fm}}(a)$ on a increases greatly with an increase in $k_{\mathrm{f}} \dot{\gamma}^{2}$, and when $k_{\mathrm{f}} \dot{\gamma}^{2} \leqq 1 \times 10^{9} / a(a$ in $\mu \mathrm{m}), \varphi_{\mathrm{fm}}(a)$ nearly equals to $\varphi_{\mathrm{v}}(a)$. (4) About $10 \%$ of the particle less than one

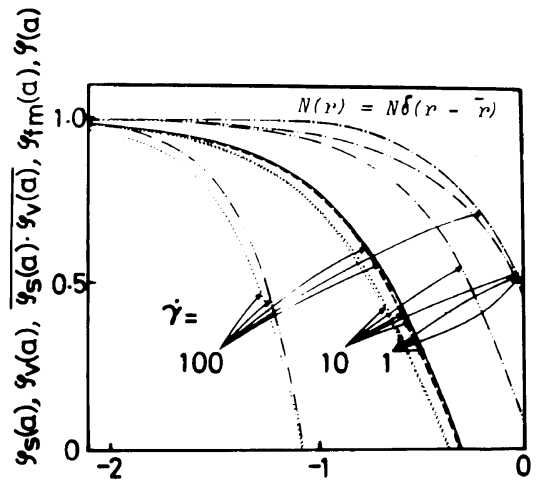

(a)

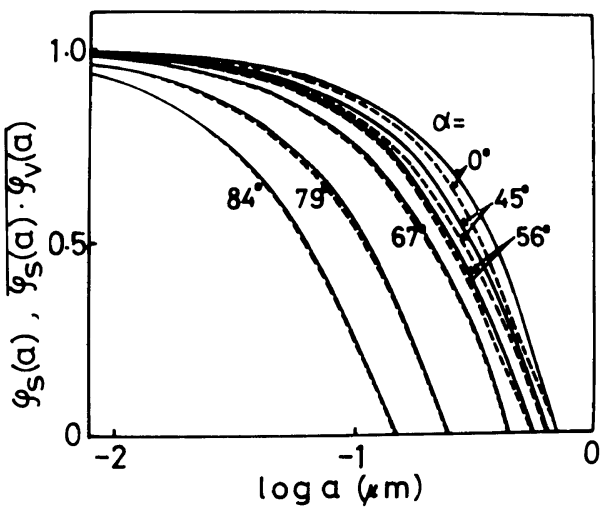

(b)

Figure 9. Particle radius dependence of $\varphi_{\mathrm{s}}(a), \varphi_{\mathrm{v}}(a)$, $\varphi_{\mathrm{fm}}(a), \overline{\varphi_{\mathrm{s}}(a) \cdot \varphi_{\mathrm{v}}(a)}$, and $\varphi(a)$ for the membrane with $N(r)=N \delta(r-r)(\bar{r}=0.776 \mu \mathrm{m})$. The numbers in (a) and (b) indicate collision angle $\alpha$ in degree and $\dot{\gamma}$ in $\mathrm{s}^{-1}$, respectively. In Figure 9(a), the full line indicates $\varphi_{\mathrm{s}}(a)$, the dot-dashed line $\varphi_{\mathrm{v}}(a)$, the double dotted chain line, $\varphi_{\mathrm{fm}}(a)$, the broken line $\overline{\varphi_{\mathrm{s}}(a) \cdot \varphi_{\mathrm{v}}(a)}$, and the dotted line, $\varphi(a)$, where, $\alpha=56^{\circ}, d=3000 \mu \mathrm{m}, k_{\mathrm{f}}=1 \times 10^{7} / a \mathrm{~s}^{2}$, and $\left(A_{\mathrm{a}, \mathrm{s}} / 3 k T\right)(\bar{a} / \bar{y})=-(a / 3) \cdot \bar{r}^{-1 / 2}$. In Figure $9(\mathrm{~b})$, full and broken lines indicate values of $\varphi_{\mathrm{s}}(a)$ and $\overline{\varphi_{\mathrm{s}}(a) \cdot \varphi_{\mathrm{v}}(a)}$, respectively.

tenth the pore size $(2 \bar{r})$ cannot pass through the pore due to the steric hindrance (i.e., $\left.\varphi_{\mathrm{s}}(a) \leqq 0.9\right)$. (5) When $\dot{\gamma}<100 \mathrm{~s}^{-1}$ and $\left(A_{\mathrm{a}, \mathrm{s}} / 3 k T\right)(\bar{a} / \bar{y})<0.1, \varphi(a, \delta)$ is governed by $\varphi_{\mathrm{s}}(a, \delta)$ and $\varphi_{\mathrm{f}}(a, \delta)$, i.e., $\varphi(a, \delta) \fallingdotseq \varphi_{\mathrm{s}}(a, \delta) \cdot \varphi_{\mathrm{f}}(a, \delta)$.

Rectangular Distribution. The intermolecular factor is negligible for large $\bar{y}$ and small $A_{\mathrm{a}, \mathrm{s}}$, and in this case eq 10 can be simplified to give 


$$
\begin{aligned}
\varphi(a) & =\varphi_{\mathrm{f}}(a, \delta) \cdot \varphi_{\mathrm{m}}(a, \delta) \frac{\int_{0}^{\infty} \varphi_{\mathrm{s}}(a, \delta) \cdot \varphi_{\mathrm{v}}(a, \delta) r^{4} N(r) \mathrm{d} r}{\int_{0}^{\infty} r^{4} N(r) \mathrm{d} r} \\
& =\varphi_{\mathrm{f}}(a, \delta) \cdot \varphi_{\mathrm{m}}(a, \delta) \cdot \frac{\frac{\varphi_{\mathrm{s}}(a) \cdot \varphi_{\mathrm{v}}(a)}{}}{}
\end{aligned}
$$

Combination of eq 58 and eq 19 yields

$$
\varphi_{\mathrm{fm}}(a)=\varphi_{\mathrm{fm}}(a, \delta)=\varphi_{\mathrm{f}}(a, \delta) \cdot \varphi_{\mathrm{m}}(a, \delta)
$$

Furthermore, if $k \leqq 0.4, \varphi_{\mathrm{v}}(a, \delta) \fallingdotseq 1.0$ holds. In the case, we obtain from eq 13 and 38 ,

$$
\varphi(a)=\varphi_{\mathrm{f}}(a, \delta) \cdot \varphi_{\mathrm{m}}(a, \delta) \cdot \varphi_{\mathrm{s}}(a)
$$

From the quantitative relationships between $\overline{\varphi_{\mathrm{s}}(a) \cdot \varphi_{\mathrm{v}}(a)}\left(\right.$ or $\left.\varphi_{\mathrm{s}}(a)\right)$ and $N(r), \varphi(a)$ can in principle be evaluated by eq 58 (or eq 60 ).

Equation 32 can be rewritten by introducing a variable $\tau$ defined by

$$
\tau=k \cdot \cos \bar{\beta}
$$

We can show that $\varphi_{\mathrm{s}}(a)$ given by eq 15 and 30 is a one parameter function of the new variable $\tau$,

$$
\varphi_{\mathrm{s}}(a)=\frac{2 \int_{0}^{\infty} \tau^{-4}\left(\sin ^{-1} \sqrt{1-\tau^{2}}-\tau \sqrt{1-\tau^{2}}\right) n(\tau) \mathrm{d} \tau}{\pi \int_{0}^{\infty} \tau^{-4} n(\tau) \mathrm{d} \tau}
$$

where

$$
\begin{aligned}
n(\tau) & =-a \cdot \cos \bar{\beta} \cdot N(r) / \tau^{2} \\
r & =a \cdot \cos \bar{\beta} / \tau
\end{aligned}
$$

Therefore, the $k$ dependence of $\varphi_{\mathrm{s}}(a, \delta)$ is comparable to its dependence on the flow rate of the filtrand in the contribution to $\varphi_{\mathrm{s}}(a)$. It should be noted that eq 56 through 64 hold independent of the form of $N(r)$.

In order to clarify the influence of the width of pore size distribution on $\varphi(a)$, the rectangular distribution shown below has been adopted.

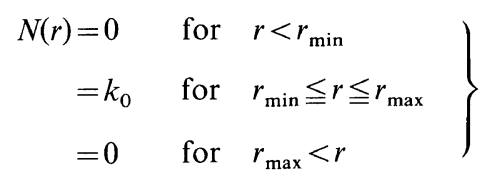

where, $r_{\min }$ and $r_{\max }$ are the minimum and maximum pore radii, respectively, and $k_{0}$ is a constant inde- pendent of $r$ and is proportional to the pore density of the membrane. Comparison of eq 65 and eq 55 reveals $k_{\mathrm{b}}=k_{0}$ and $b=0$; i.e., eq 65 belongs to the family of exponential function.

The Poiseuille law indicates that membranes with the same $\left(\bar{r}_{3} \cdot \bar{r}_{4}\right)^{1 / 2}$ value and the same $P_{\mathrm{r}}$ values should given the same filtration rate. Hence,

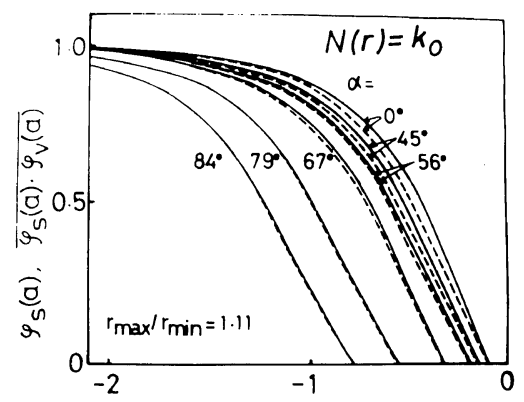

(a)

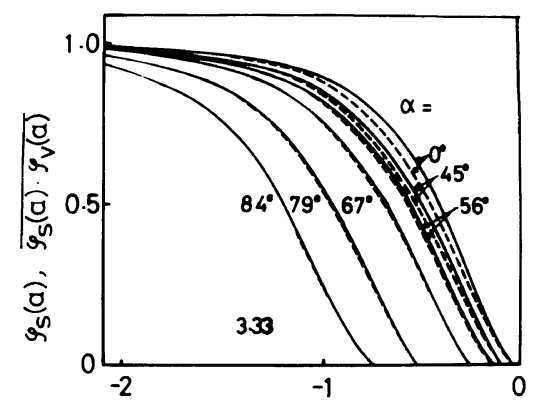

(b)

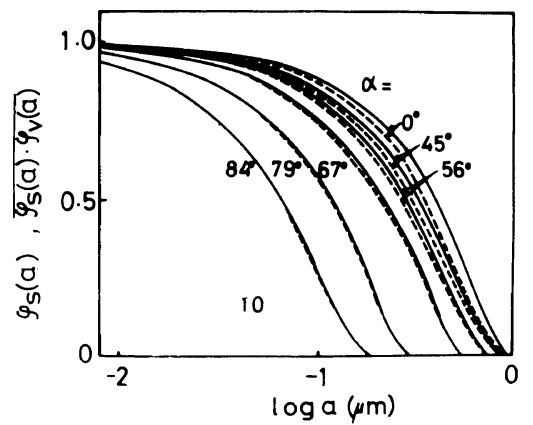

(c)

Figure 10. Particle radius dependence of $\varphi_{\mathrm{s}}(a)$ and $\overline{\varphi_{\mathrm{s}}(a) \cdot \varphi_{\mathrm{v}}(a)}$ for the membrane with rectangular distribution of $N(r)$ with $\left(\bar{r}_{3} \cdot \bar{r}_{4}\right)^{1 / 2}=0.776 \mu \mathrm{m}$ : Full and broken lines indicate the values of $\varphi_{\mathrm{s}}(a)$ and $\overline{\varphi_{\mathrm{s}}(a) \cdot \varphi_{\mathrm{v}}(a) \text {, }}$ respectively. The numbers in this figure denote $\alpha$ values in degree. Figures $\mathrm{a}, \mathrm{b}$, and $\mathrm{c}$ correspond to the membranes with $r_{\max } / r_{\min }=0.1,0.3$, and 0.9 , respectively. 
$\left(\bar{r}_{3} \cdot \bar{r}_{4}\right)^{1 / 2}=0.776 \mu \mathrm{m}$, the same as that used in the previous section, was also utilized for the further study.

Figure 10 shows the particle size dependence of $\varphi_{\mathrm{s}}(a)$ (full line) and $\overline{\varphi_{\mathrm{s}}(a) \cdot \varphi_{\mathrm{v}}(a)}$ (broken line) for various collision angles $\alpha$. The membrane was assumed to have $\left(\bar{r}_{3} \cdot \bar{r}_{4}\right)^{1 / 2}=0.776 \mu \mathrm{m}$ and the ratios between the maximum and minimum pore size $r_{\max } / r_{\min }$ were taken to be 10 (Figure 10a), 3.33 (Figure 10b), and 1.11 (Figure 10c). For $r_{\text {max }} / r_{\min }=1.11$ (Figure 10c), $\varphi_{\mathrm{s}}(\mathrm{a})$ and $\overline{\varphi_{\mathrm{s}}(a) \cdot \varphi_{\mathrm{v}}(a)}$ are nearly equal to those obtained with $N(r)=N \delta(r-\bar{r})$ (see Figure 9). Both $\varphi_{\mathrm{s}}(a)$ and $\overline{\varphi_{\mathrm{s}}(a) \cdot \varphi_{\mathrm{v}}(a)}$, for a given $a$ value, decrease gradually with an decrease in $r_{\max } / r_{\min }$. This indicates that the broadening of pore size distribution allows $\varphi(a)$ to increase.

Figure 11 shows the changes in $\varphi_{\mathrm{v}}(a)$ and $\overline{\varphi_{\mathrm{s}}(a) \cdot \varphi_{\mathrm{v}}(a)} / \varphi_{\mathrm{s}}(a)\left(=\overline{\bar{\varphi}}_{\mathrm{v}}(a)\right)$ with $r_{\text {max }} / r_{\text {min }}$ for various $\alpha$ values. In this case, $a=0.40 \mu \mathrm{m}$ and $\left(\bar{r}_{3} \cdot \bar{r}_{4}\right)^{1 / 2}=0.776 \mu \mathrm{m}$ were assumed. Both $\varphi_{\mathrm{v}}(a)$ and $\overline{\bar{\varphi}}_{\mathrm{v}}(a)$ values increase with $r_{\text {max }} / r_{\text {min }}$, approaching an asymptotic value at $r_{\max } / r_{\min }=3.0$. The asymptotic values of $\overline{\bar{\varphi}}_{\mathrm{v}}(a)$ are larger for larger $\alpha \cdot \overline{\bar{\varphi}}_{\mathrm{v}}(a)$ is always larger than $\varphi_{\mathrm{v}}(a)$. As is obvious from eq 58 and 60 , $\bar{\varphi}_{\mathrm{v}}(a)$ represents the contribution of the viscous factor toward $(a)$. A larger $\overline{\bar{\varphi}}_{\mathrm{v}}(a)$ means a lesser contribution toward $\varphi(a)$. Thus the viscous factor is less predominant for wider pore size distribution (i.e., $r_{\max } / r_{\min }$ ) and larger $\alpha$. This can be explained as follows: Since the power of $r$ in eq 18 is four, the pore with a larger radius contributes to a greater $\overline{\varphi_{\mathrm{s}}(a) \cdot \varphi_{\mathrm{v}}(a)}$ values, and eq 33 indicates that $\varphi_{\mathrm{v}}(a, \delta)$ for a larger pore is closer to unity, so that $\bar{\varphi}_{\mathrm{v}}(a)$ increase with an increase in $r_{\max } / r_{\min }$.

Pore Size Distribution with a Long Tail. Next we consider the case in which pore size distribution $N(r)$ can be expressed by an exponential function with either $b=4$ or $b=-4$ in eq 55. The $N(r) v s . r$ curve corresponding to $b=4$ has a tail at small $r$ and the $N(r) v s$. $r$ curve for $b=-4$ has a tail at large $r$. If we specify $\left(\bar{r}_{3} \cdot \bar{r}_{4}\right)^{1 / 2}$ and $r_{\min }$, the $r_{\max }$ values of the two $N(r)$ functions can be determined theoretically.

Figures $12 \mathrm{a}$ and $\mathrm{b}$ show $\varphi_{\mathrm{s}}(a)$ and $\overline{\varphi_{\mathrm{s}}(a) \cdot \varphi_{\mathrm{v}}(a)} v \mathrm{~s}$. $a$ curves of the membrane with $\left(\bar{r}_{3} \cdot \bar{r}_{4}\right)^{1 / 2}=0.776 \mu \mathrm{m}$ and $b=4$ and -4 , respectively. For $b=4, N(r)=0$ holds in the ranges of $r>0.886 \mu \mathrm{m}$ and $r<10^{-4} \mu \mathrm{m}$, and for $b=-4, N(r)=0$ for $r>12 \mu \mathrm{m}$ and

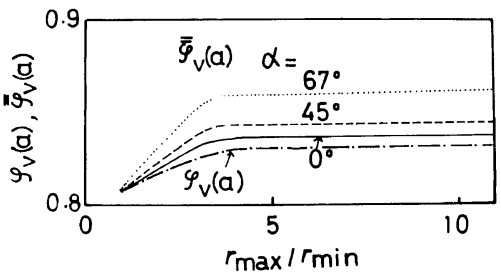

Figure 11. Contributions of the viscosity factor toward $\varphi(a)$, and are represented by $\varphi_{\mathrm{v}}(a)$ and $\bar{\varphi}_{\mathrm{v}}(a)_{\mathrm{s}}$, as a function of $r_{\max } / r_{\min }$ and $\alpha$. The numbers denote value of $\alpha$ in degrees. $a=0.410 \mu \mathrm{m}$ and $\left(\bar{r}_{3} \cdot \bar{r}_{4}\right)^{1 / 2}=0.776 \mu \mathrm{m}$.

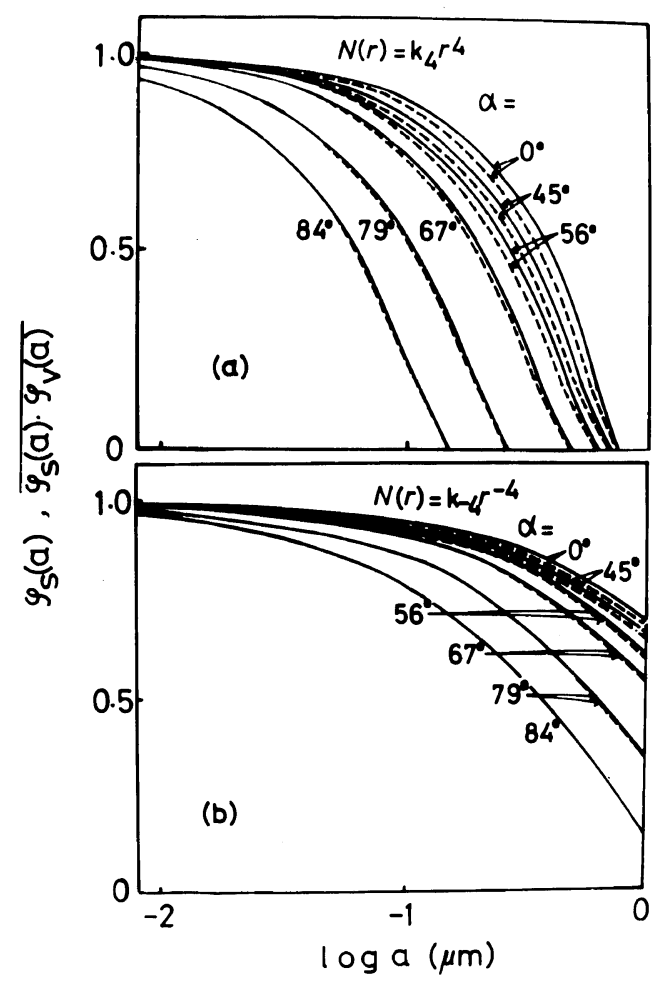

Figure 12. $\varphi_{\mathrm{s}}(a)$ vs. aand $\overline{\varphi_{\mathrm{s}}(a) \cdot \varphi_{\mathrm{v}}(a)}$ vs. $a$ curves for membranes with the same mean pore radii $\left(\bar{r}_{3} \cdot \bar{r}_{4}\right)^{1 / 2}=0.776 \mu \mathrm{m}$ and different pore size distributions (a) $N(r)=k_{4} r^{4}(b=4)$, (b) $N(r)=k_{-4} r^{-4}(b=-4)$. Full and broken lines indicate the values of $\varphi_{\mathrm{s}}(a)$ and $\varphi_{\mathrm{s}}(a) \cdot \varphi_{\mathrm{v}}(a)$, respectively. The number denote $\alpha$ values in degrees.

$r<0.05 \mu \mathrm{m} . \varphi_{\mathrm{s}}(a)$ as well as $\overline{\varphi_{\mathrm{s}}(a) \cdot \varphi_{\mathrm{v}}(a)}$ for $b=4$ are smaller than those for $b=-4$ at any $\alpha$. The slope of the curve, $|\mathrm{d} \varphi(a) / \mathrm{d} \ln a|$, for $b=4$ is larger than that for $b=-4$. The viscous factor represented by $\overline{\bar{\varphi}}_{\mathrm{v}}(a)$ is more evident in the case of $b=4$ than $b=-4$. 
In summary, the steric and viscous factors represented by $\overline{\varphi_{\mathrm{s}}(a) \cdot \varphi_{\mathrm{v}}(a)}$ play a less important role in $\varphi(a)$ as in the width of the pore size distribution increases. In the theories proposed so far, only the steric factor $^{5}$ (see eq 5) or the steric and viscous factors $^{6}$ (see eq 6) on $\varphi(a)$ was discussed without taking into account of the pore size distribution. A comparison of the theories was made with the experiments by taking appropriate values for $r$. From theoretical point of view, there is the problem as to what mean pore size should be used for $r$ in eq 5 and 6 . This cannot be ignored since the mean pore size changes in principle depending on the method of measurement as long as the distribution of pore size is significant. Anyhow, the pore size distribution contributes to $\varphi(a)$ considerably.

\section{Permselectivity of Porous Membrane (Calculation)}

Permselectivity with respect to particle size, as represented by $\mathrm{d} \varphi(a) / \mathrm{d} a$, changes with mean pore size. Therefore, in the case of $N(r)=N \delta(r-\bar{r})$, $\mathrm{d} \varphi(a) / \mathrm{d} k(k=a / r)$ is preferable for eliminating the contribution of mean pore size. For an actual membrane with broad pore size distribution, $k$ should be replaced by $a /\left(\bar{r}_{3} \cdot \bar{r}_{4}\right)^{1 / 2}$. We define, for convenience, the permselectivity power $P_{\mathrm{se}}$ by,

$$
P_{\mathrm{se}}=-\left(\bar{r}_{3} \cdot \bar{r}_{4}\right)^{1 / 2}(\mathrm{~d} \varphi(a) / \mathrm{d} a)_{\varphi=0.5}
$$

where the suffix $\varphi=0.5$ indicates the value at $\varphi=0.5^{\circ} P_{\text {se }}$ can be evaluated from the experimental $\varphi(a) v s$. $a$ curves and the value of $\left(\bar{r}_{3} \cdot \bar{r}_{4}\right)^{1 / 2}$.

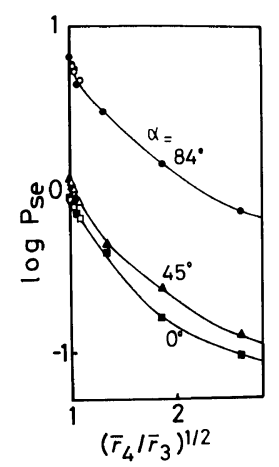

Figure 13. Dependence of permselectivity $\dot{P}_{\text {se }}$ on $\bar{r}_{4} / \bar{r}_{3}$. The numbers denote the collision angles $\alpha$ in degree. $\boldsymbol{\Delta}, \boldsymbol{\square}$, model membranes with $\left(\bar{r}_{3} \cdot \bar{r}_{4}\right)^{1 / 2}=0.776 \mu \mathrm{m}$ and $b=-4 \sim 5$ in $N(r)=k_{\mathrm{b}} r^{\mathrm{b}} ; \bigcirc, \triangle, \square$, actual membranes $\mathrm{Nu} 0.8, \mathrm{Nu} 0.6$ and SF 0.3, Circular, triangle, and regular square marks indicate values of $P_{\text {se }}$ at angles $84^{\circ}$, $45^{\circ}$, and $0^{\circ}$, respectively.
Figure 13 shows the $\bar{r}_{4} / \bar{r}_{3}$ dependence of $P_{\text {se }}$ for hypothetical membranes with $b=-4 \sim 5$ (see eq 55) and for the actual membranes such as $\mathrm{Nu} 0.8, \mathrm{Nu}$ 0.6 , and SF 0.3 . All the $\left(\bar{r}_{3} \cdot \bar{r}_{4}\right)^{1 / 2}$ values of the hypothetical membrane are $0.776 \mu \mathrm{m}$. Plots of $P_{\text {se }}$ vs. $\bar{r}_{4} / \bar{r}_{3}$ for various membranes at a given $\alpha$ can be represented by a single curve, independent of $N(r)$ and, thus, of $\left(\bar{r}_{3} \cdot \bar{r}_{4}\right)^{1 / 2} . P_{\text {se }}$ thus increases with a decrease in $\bar{r}_{4} / \bar{r}_{3}$ and with an increase in $\alpha$.

In order to obtain a membrane having both large permeability and permselectivity, we must design the membrane by which large absolute values of $N(r)$ and large $\left(\bar{r}_{3} \cdot \bar{r}_{4}\right)^{1 / 2}$ and small $\bar{r}_{4}$ values can be obtained. In addition, the choice of filtration conditions which give a large $\alpha$ value (for example, large $\dot{\gamma}$ is very important in achieving this purpose.

\section{Comparison between Theoretical and Experimental $\varphi($ a) Values}

The $N(r)$ curve determined for SF 0.3 by using the mercury intrusion method is shown in Figure 14a. Figure $14 \mathrm{~b}$ shows the relation of $\varphi_{\mathrm{s}}(a)$ and $\overline{\varphi_{\mathrm{s}}(a) \cdot \varphi_{\mathrm{v}}(a)}$ to $\log \left(a /\left(\bar{r}_{3} \cdot \bar{r}_{4}\right)^{1 / 2}\right)$ obtained by putting this $N(r)$ into eq 15 and 18 . Figures $15 \mathrm{a}$ and $15 \mathrm{~b}$ demonstrate similar results for $\mathrm{Nu} 0.8$. Inspection of Figures 8, 9, 11, 14b and $15 \mathrm{~b}$ leads to the conclusion that the long tail of $N(r)$ at larger $r$ produces the tail of the corresponding $\varphi_{\mathrm{s}}(a)$ (or $\left.\overline{\varphi_{\mathrm{s}}(a) \cdot \varphi_{\mathrm{v}}(a)}\right) \quad v s . \quad a /\left(\bar{r}_{3} \cdot \bar{r}_{4}\right)^{1 / 2}$ curve in the larger $a /\left(\bar{r}_{3} \cdot \bar{r}_{4}\right)^{1 / 2}$ region. Figure 16 shows a plot of the permeability coefficient $P_{\mathrm{e}}$ for a $0.05 \mathrm{wt} \%$ aq solution of dextran against the pressure difference $\Delta P$ for SF 0.2. If concentration (gel) polarization occurs, $P_{\mathrm{e}}$ must decrease significantly with an increase in $\Delta P$, as is always observed in reverse osmosis. ${ }^{19}$ However, in this case, $P_{\mathrm{e}}$ is evidently almost independent of $\Delta P$. When the solution is not filtrated in advance through $\mathrm{Nu} 0.8$ under the condition of $\dot{\gamma}=0, P_{\mathrm{e}}$ decreases with filtration time. Once it has decreased, $P_{\mathrm{e}}$ can not be recovered by repetition of filtrating, followed by standing without filtration under $\Delta P=0$. These facts contradict the theoretical prediction of the concentration polarization hypothesis. ${ }^{19}$ Thus the contribution of concentration polarization toward $\varphi(a)$, if it exists, can be neglected in our experiment. The change in $\varphi(a)$ with the parameter $\alpha$ (eq 20) is shown in Figure 17, for the filtration of aq solutions of urea and of dextrans by SF 0.2 . Here, $\alpha$ was calculated by putting the 


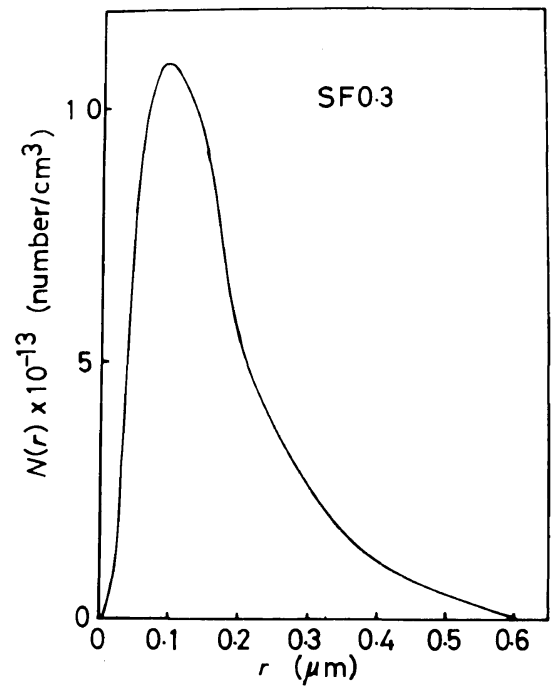

(a)

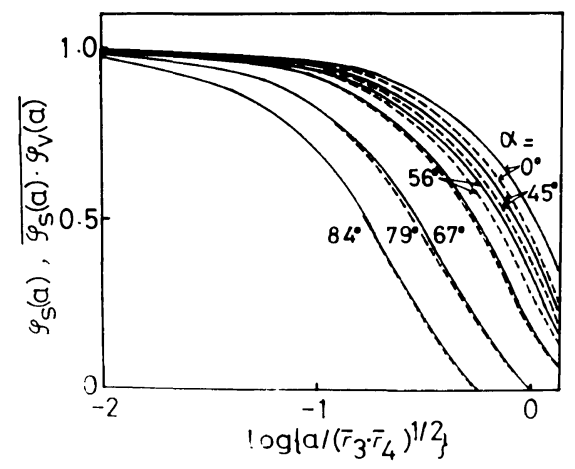

(b)

Figure 14. Experimental pore size distribution curve and theoretical $\varphi_{\mathrm{s}}(a)$ and $\overline{\varphi_{\mathrm{s}}(a) \cdot \varphi_{\mathrm{v}}(a)}$ vs. $a /\left(\bar{r}_{3} \cdot \bar{r}_{4}\right)^{1 / 2}$ curve for SF 0.3: (a), experimental $N(r)$; (b), $\varphi_{\mathrm{s}}(a)$ (full line and $\overline{\varphi_{\mathrm{s}}(a) \cdot \varphi_{\mathrm{v}}(a)}$ (broken line). The numbers indicate collision angles $\alpha$ in degree. $\left(\bar{r}_{3} \cdot \bar{r}_{4}\right)^{1 / 2}=0.18 \mu \mathrm{m}$.

observed $P_{\mathrm{r}}(=0.76)$ and $r\left(=\left(\bar{r}_{3} \cdot \bar{r}_{4}\right)^{1 / 2}=0.100 \mu \mathrm{m}\right)$ for SF 0.2 , and the experimental conditions for $d, \bar{u}$ and $v_{\mathrm{f}}$ into eq $20 . \alpha$ is allowed to vary with external conditions such as $v_{\mathrm{f}}$, which is proportional to $\Delta P, d$, and $\dot{\gamma}(=6 \bar{u} / d)$. The circles are experimental data points. The numbers for dextran in this figure denote the weight-average molecular weights $M_{w}$ of the sample. Evidently, $\varphi(a)$ decreases monotonicall with an increases in $\alpha$ and $M_{w}$. We can reduce $\varphi(a)$ vs. $\alpha$ plots for a wide variety of $\dot{\gamma}\left(10-70 \mathrm{~s}^{-1}\right)$ and $\Delta P(10-500 \mathrm{mmHg})$ to a single master curve.

The particle diameter $2 a$ of urea was estimated

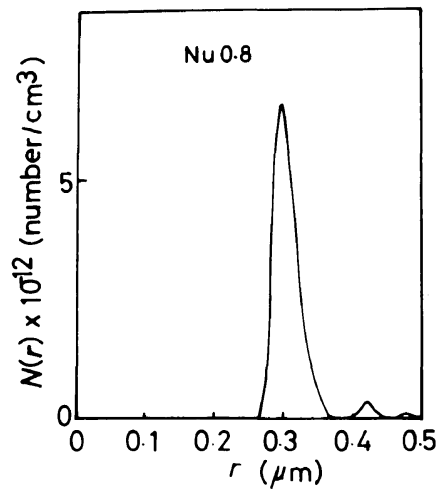

(a)

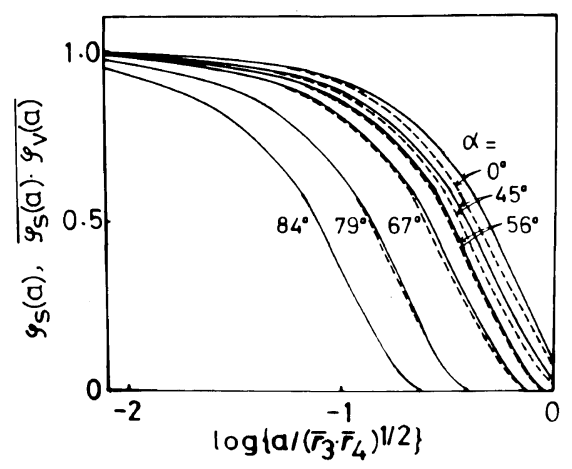

(b)

Figure 15. Experimental pore size distribution curve and theoretical $\varphi_{\mathrm{s}}(a)$ and $\overline{\varphi_{\mathrm{s}}(a) \cdot \varphi_{\mathrm{v}}(a)}$ vs. $a /\left(\bar{r}_{3} \cdot \bar{r}_{4}\right)^{1 / 2}$ curves for $\mathrm{Nu}$ 0.8: (a), experimental $N(r) ;(\mathrm{b}), \varphi_{\mathrm{s}}(a)$ (full line) and $\overline{\overline{\varphi_{\mathrm{s}}}(a) \cdot \varphi_{\mathrm{v}}(a)}$ (broken line). The numbers indicate collision angles $\alpha$ in degrees. $\left(\bar{r}_{3} \cdot \bar{r}_{4}\right)^{1 / 2}=0.400 \mu \mathrm{m}$.

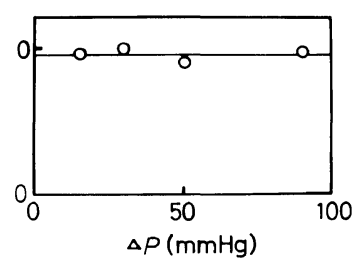

Figure 16. Dependence of the experimental permeability coefficient $P_{\mathrm{e}}$ on the applied pressure difference $\Delta P$ for SF 0.2: solution (filtrand), $0.05 \mathrm{wt} \%$ aq solution of dextran $\left(M_{w}=1.0 \times 10^{5}\right)$.

from its molecular structure to be $0.4 \mathrm{~nm}$ and those of dextran were assumed to be equal to the mean square diameter of gyration $2\left\langle S_{0}\right\rangle^{1 / 2}$ in a $\theta$ solvent and calculated from

$$
2 a=2\left\langle S_{0}\right\rangle^{1 / 2}=1.02 \times 10^{-4} \mathrm{M}^{1 / 2} \quad(\text { in } \mu \mathrm{m})(67)
$$




\section{K. KAMIDE and S. MANABE}
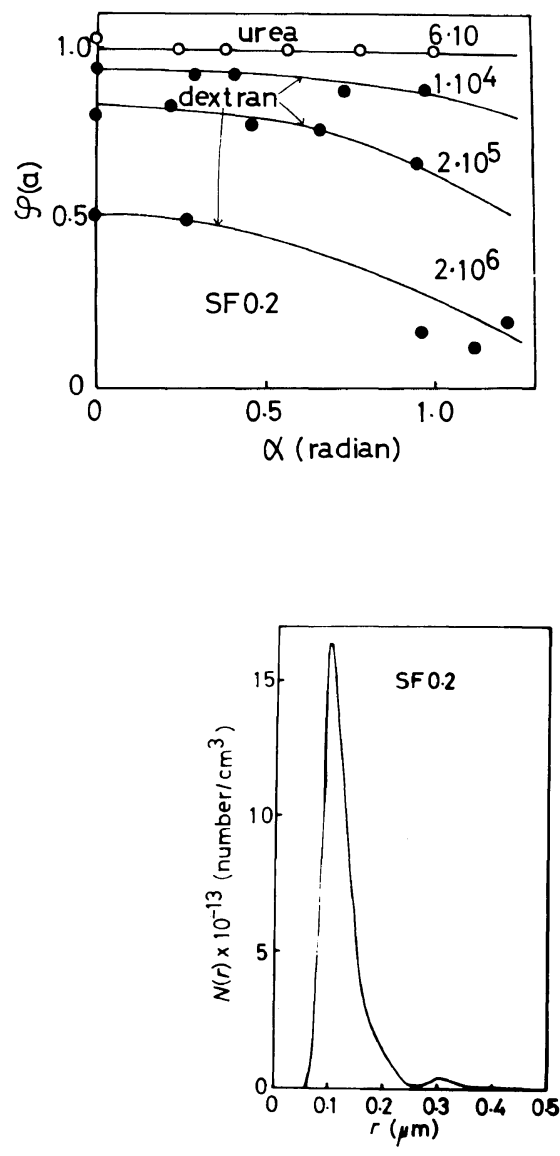

(a)
Figure 17. Changes in experimental $\varphi(a)$ of certain model substance for SF 0.2 under filtration condition (i.e., $\Delta P$ and $\gamma$ ). $\alpha$ is calculated by putting the observed values of $P_{\mathrm{r}}(=0.76), d(1.0 \mathrm{~mm}), r \quad\left(=\left(\bar{r}_{3} \cdot \bar{r}_{4}\right)^{1 / 2}=\right.$ $0.110 \mu \mathrm{m})$ for SF 0.2 and the experimental values of $\bar{u}$ and $v_{\mathrm{f}}$ into eq 20 . The numbers denote weight-average molecular weights.

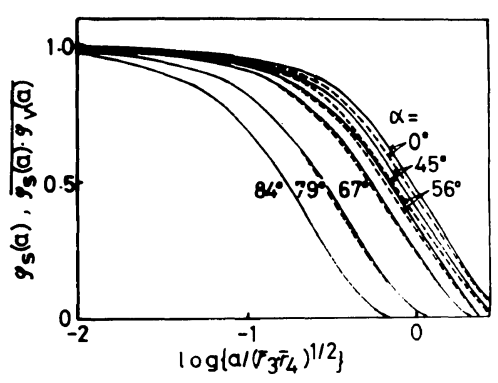

(b)

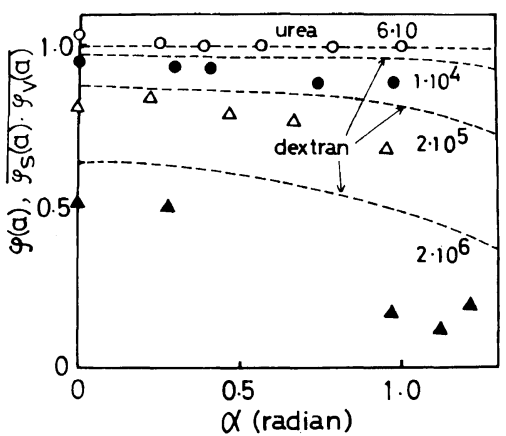

(c)

Figure 18. Comparison between experimental and theoretical $\varphi(a)$ of various model substances for SF 0.2 : (a), observed $N(r)$; (b), theoretical values of $\varphi_{\mathrm{s}}(a)$ (full line) and $\overline{\varphi_{\mathrm{s}}(a) \cdot \varphi_{\mathrm{v}}(a)}$ (broken line) calculated by using the $N(r)$. The numbers in Figure 18b indicate the collision angle $\alpha$ in degree; (c), plots of observed $\varphi(a)$ against $\alpha$ and calculated $\overline{\varphi_{\mathrm{s}}(a) \cdot \varphi_{\mathrm{v}}(a)}$ values (broken line) reploted from Figure $18 \mathrm{~b}$, the numbers in this figure indicate molecular weight; $\bigcirc$, observed $\varphi(a)$ for urea; $\bigcirc$, dextran with $M_{w}=1 \times 10^{4} ; \triangle$, dextran with $M_{w}=2 \times 10^{5} ; \boldsymbol{\Delta}$, dextran with $M_{w}=2 \times 10^{6}$. 


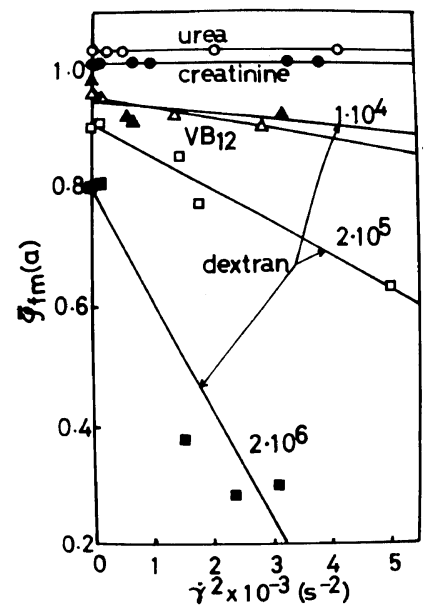

Figure 19. Plots of $\overline{\bar{\varphi}}_{\mathrm{fm}}(a)$ for SF 0.2 against $\dot{\gamma}^{2} \cdot \bar{\varphi}_{\mathrm{fm}}(a)$ is estimated from Figure 18c. $\dot{\gamma}$ is calculated from the value of $\bar{u}$ by using the relation $\dot{\gamma}=6 \bar{u} / d$.

where, $M_{w}$ is adopted in place of $M$.

Figure 18a shows the observed pore size distribution function $N(r)$ for SF 0.2 , and Figure 18b, the theoretical curves for $\varphi_{\mathrm{s}}(a)$ (full line) and $\overline{\varphi_{\mathrm{s}}(a) \cdot \varphi_{\mathrm{v}}(a)}$ (broken line) obtained by using this $N(r)$. We calculated theoretical relations between $\overline{\varphi_{\mathrm{s}}(a) \cdot \varphi_{\mathrm{v}}(a)}$ and $a /\left(\bar{r}_{3} \cdot \bar{r}_{4}\right)^{1 / 2}$ for solutions filtered through SF 0.2. The results are shown as broken lines in Figure 18c. Experimental $\varphi(a)$ data points are in fairly good agreement with theoretical $\overline{\varphi_{\mathrm{s}}(a) \cdot \varphi_{\mathrm{v}}(a)}$ for urea, and for three dextrans.

Figure 19 shows the $\dot{\gamma}^{2}$ dependence of $\overline{\bar{\varphi}}_{\mathrm{fm}}(a)$ estimated from the observed $\varphi(a)$ and by using eq 19 the calculated $\overline{\varphi_{\mathrm{s}}(a) \cdot \varphi_{\mathrm{v}}(a)}$ values shown by the full lines in Figure 18. Combination of eq 59 and 52 leads us to give

$$
\overline{\bar{\varphi}}_{\mathrm{fm}}(a)=\left\{1-k_{\mathrm{f}} \dot{\gamma}^{2}(2 a / d)^{2.84}\right\}\left\{1+\left(A_{\mathrm{a}, \mathrm{s}} / 3 k T\right)(\bar{a} / \bar{y})\right\}
$$

Equation 68 predicts that $\overline{\bar{\varphi}}_{\mathrm{fm}}(a)$ is a linear function of $\dot{\gamma}^{2}$ and in fact the experimental data points in Figure 19 can be fitted approximately to a straight line.

In Table II are summarized the values of $k_{\mathrm{f}}$ value and $\left(A_{\mathrm{a}, \mathrm{s}} / 3 k T\right)(\bar{a} / \bar{y})$ value, experimentally evaluated from the slopes and intercepts of the full lines in Figure 19 for various substances filtered through SF 0.2 . Both $k_{\mathrm{f}}$ and $\left(A_{\mathrm{a}, \mathrm{s}} / 3 k T\right)(\bar{a} / \bar{y})$ depend on the
Table II. Experimental values of $k_{\mathrm{f}}$ and $\left(A_{\mathrm{a}, \mathrm{s}} / 3 k T\right)(\bar{a} / \bar{y})$ of various substances for SF 0.2

\begin{tabular}{llc}
\hline \multicolumn{1}{c}{ Substance } & \multicolumn{1}{c}{$k_{\mathrm{f}} / \mathrm{s}^{2}$} & $\left(A_{\mathrm{a}, \mathrm{s}} / 3 k T\right)(\bar{a} / \bar{y})$ \\
\hline Urea & 0 & 0.03 \\
Creatinine & 0 & 0.01 \\
VB $_{12}$ & $3.42 \times 10^{11}$ & -0.184 \\
Dextran $M_{w}=1 \times 10^{4}$ & $4.78 \times 10^{10}$ & -0.049 \\
Dextran $M_{w}=20 \times 10^{4}$ & $6.87 \times 10^{8}$ & -0.063 \\
Dextran $M_{w}=200 \times 10^{4}$ & $4.03 \times 10^{8}$ & -0.132 \\
\hline
\end{tabular}

chemical structure of the molecule making up a particle. When the chemical structure is similar as in this series of dextrans with different $M_{w}, k_{\mathrm{f}}$ decreases and the absolute value of $\left(A_{\mathrm{a}, \mathrm{s}} / 3 k T\right)(\bar{a} / \bar{y})$ increases with $M_{w}$. the former may be interpreted on the basis of the molecular weight dependence of the diffusion coefficient $D_{\mathrm{a}}$ as indicated by eq 44 , and the latter may be interpreted in terms of the molecular weight dependence of the particle radius $a$ as indicated by eq 48 . The distinction between positive and negative values of $\left(A_{\mathrm{a}, \mathrm{s}} / 3 k T\right)(\bar{a} / \bar{y})$ is significant in a qualitative discussion concerning the molecular interaction. For example, from the view point of molecular interaction between solute particle and membrane, a cellulose acetate membrane has a tendency to allow urea and creatine molecules to pass through its pore, much more easily than do $\mathrm{VB}_{12}$ and dextran.

Figure 20a shows the experimental $N(r)$ for $\mathrm{Nu}$ 0.08 , and Figure $20 \mathrm{~b}$ shows the plot of the theoretical $\varphi_{\mathrm{s}}(a)$ (full line) and $\overline{\varphi_{\mathrm{s}}(a) \cdot \varphi_{\mathrm{v}}(a)}$ (broken line) as a function of $a /\left(\bar{r}_{3} \cdot \bar{r}_{4}\right)^{1 / 2}$ for $\mathrm{Nu} 0.08$. These two curves nearly coincide with each other. In Figure $20 \mathrm{c}$ are shown the $\alpha$ dependence of experimental $\varphi(a)$ data points and the theoretical $\overline{\varphi_{\mathrm{s}}(a) \cdot \varphi_{\mathrm{v}}(a)}$ curvies. The agreement between these $\varphi(a)$ and $\overline{\varphi_{\mathrm{s}}(a) \cdot \varphi_{\mathrm{v}}(a)}$ is not as good as in the case of SF 0.2 , but the tendency of $\varphi(a)$ to decrease with $\dot{\gamma}$ is significant, as the theory predicts. Since $v_{\mathrm{f}}$ is proportional to $r^{4}$ for a given $\Delta P$, eq 34 indicates that $\bar{y}$ is proportional to $r^{2.5}$ at a fixed value of $P_{\mathrm{r}}$. Then, $\varphi_{\mathrm{m}}(a, \delta)$ given by eq 51 shows $r$ dependence in the region of small $r$. In this case $\bar{y}$ is very small, and makes eq 59 and eq 19 inapplicable. if we take this $r$ dependence of $\varphi_{\mathrm{f}}(a, \delta)$ into account, the following equation is more reliable than eq 68 , 


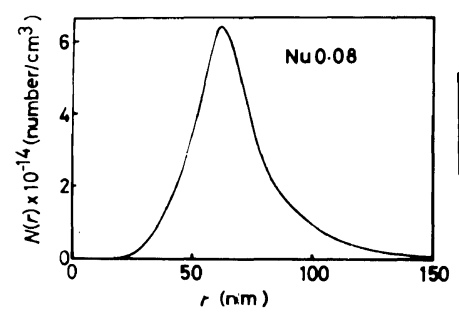

(a)

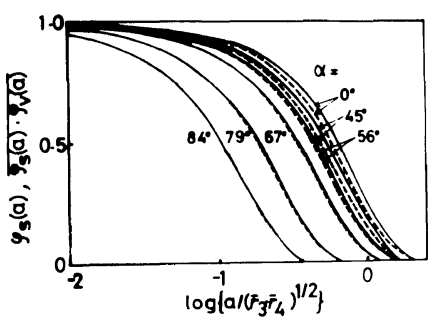

(b)

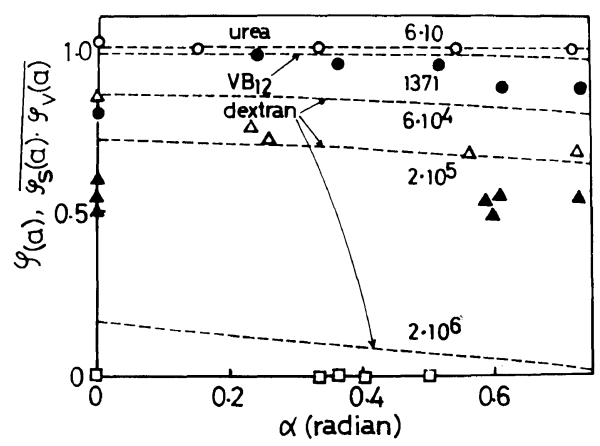

(c)

Figure 20. Comparison between observed values of $\varphi(a)$ and calculated value of $\varphi_{\mathrm{s}}(a) \cdot \varphi_{\mathrm{v}}(a)$ of some model substances in aq solution for Nu 0.08: (a), observed $N(r)$; (b), theoretical values of $\varphi_{\mathrm{s}}(a)$ (full line) and $\overline{\varphi_{\mathrm{s}}(a) \cdot \varphi_{\mathrm{v}}(a)}$ (broken line); (c), observed $\varphi(a)\left(\bigcirc\right.$, urea; $\boldsymbol{O}, \mathrm{VB}_{12} ; \triangle, \boldsymbol{\Delta}, \square$, dextran with $M_{w}=6 \times 10^{4}$, $2 \times 10^{5}$, and $2 \times 10^{6}$, respectively) and theoretical value of $\overline{\varphi_{\mathrm{s}}(a) \cdot \varphi_{\mathrm{v}}(a)}$ (broken line). The number in Figure $20 \mathrm{~b}$ indicate collision angle $\alpha$ in degree.

$$
\overline{\bar{\varphi}}_{\mathrm{fm}}(a)=\overline{\bar{\varphi}}_{\mathrm{m}}(a)-k_{\mathrm{f}}(2 a / d)^{2.84} \dot{\gamma}^{2}
$$

where $\bar{\varphi}_{\mathrm{m}}(a)$ is an adjusting parameter evaluated experimentally from $\varphi_{\mathrm{fm}}(a)$ at $\dot{\gamma}=0$. By applying eq 69 to the data in Figure 20c, we obtain $k_{\mathrm{f}}$ of the $\mathrm{Nu} 0.08$ membrane to be $0,5 \times 10^{11}, 8 \times 10^{10}$, and $2 \times 10^{8}\left(\mathrm{~s}^{2}\right)$ for urea, $\mathrm{VB}_{12}$ and two dextrans of $M_{w}=6 \times 10^{4}$, and $2 \times 10^{5}$, respectively. These values are comparable to those for SF $0.2 \mathrm{mem}$ brane in Table II in their relative order.

The observed $\varphi(a)$ values of various substances for SF 0.2 and Nu 0.08 decrease with an increase in $\alpha$ if the molecular weight of a particle is larger than at least $10^{3}$. This fact has been explained in part by the concentration polarization hypothesis, which unfortunately is inadequate for explaining the pressure dependence of the filtration rate observed in this study (see Figure 16). It is clear that the shear rate $\dot{\gamma}$ dependence of $\varphi(a)$ can only be interpreted by the present theory, in which the $\alpha$ dependence of $\varphi_{\mathrm{s}}(a)$, and $\overline{\varphi_{\mathrm{f}}(a) \cdot \varphi_{\mathrm{m}}(a)}$ is taken into account.
Our permselectivity theory has been applied to estimating the mean pore size of a regenerated cellulose membrane "Cuprophan," for which the previous methods ${ }^{23,24}$ were not adequate since the pore size of the membrane is smaller than $10 \mathrm{~nm}$.

The shear rate $\dot{\gamma}$ dependence of $\varphi(a)$ for aq solution of some model substances is shown in Figure 21. A slight decrease in $\varphi(a)$ with an increase in $\dot{\gamma}$ is observed as expected from the theory (see eq $53)$, except for urea $(\varphi(a)=1.0)$ and dextran $\left(M_{w}=2 \times 10^{6}\right)(\varphi(a)=0.00)$. The experimental values of $\varphi(a)$ at $\dot{\gamma}=0$ are plotted as a function of $\log a$ ( $a$; particle radius) in Figure 22. The Best fit of the theoretical $\varphi(a) v s$. a curve to the experimental points was achieved by $N(r)=k_{-4} r^{-4}$ with $r_{\max }=20 \mathrm{~nm}, \bar{r}_{4} / \bar{r}_{3}=26.5$, and $\left(\bar{r}_{3} \cdot \bar{r}_{4}\right)^{1 / 2}=3.8 \mathrm{~nm}$, and is shown as a broken line in Figure 21. Since $\varphi(a)=\overline{\varphi_{\mathrm{s}}(a) \cdot \varphi_{\mathrm{v}}(a)}$ was assumed in this case (that is, $\varphi_{\mathrm{m}}(a, \delta)=1$ was assumed in eq 58), the actual values of $\left(\bar{r}_{3} \cdot \bar{r}_{4}\right)^{1 / 2}$ and $r_{\max }$ might be slightly larger than the values obtained here. 


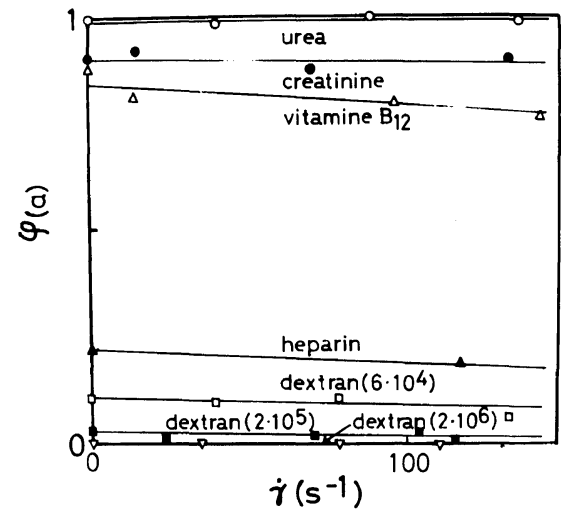

Figure 21. Dependence of experimental $\varphi(a)$ on shear rate $\dot{\gamma}$ of some model substances for Cuprophan: The numbers in the parentheses for dextran indicate weightaverage molecular weight.

The value of $7.6 \mathrm{~nm}$ for the pore size is about twice as large as that in the literature $(3.1 \mathrm{~nm})$ for Cuprophan $^{21}$ and is nearly equal to the literature value $(7.3 \mathrm{~nm})$ for cellophane. ${ }^{1}$ We have derived the relation between porosity of membrane $P_{\mathrm{r}}$, water permeability coefficient $P_{\mathrm{e}}$ and mean pore radius $\left(\bar{r}_{3} \cdot \bar{r}_{4}\right)^{1 / 2}$. by using Poiseuille law as follows:

$$
P_{\mathrm{r}}=8 \eta P_{\mathrm{e}} /\left(\bar{r}_{3} \cdot \bar{r}_{4}\right)
$$

where $\eta$ is the viscosity of water. $P_{\mathrm{e}}$ for Cuprophan was evaluated from an ultrafiltration experiment to be $1.14 \times 10^{-4} \mathrm{~cm}^{2} \mathrm{~s}^{-1} \mathrm{mmHg}^{-1}$. If we put $P_{\mathrm{e}}=1.14 \times 10^{-4}, \quad\left(\bar{r}_{3} \cdot \bar{r}_{4}\right)^{1 / 2}=3.8 \mathrm{~nm}$ and $\eta=0.80$ centipoise (at $38^{\circ} \mathrm{C}$ ) into eq 70 , we obtain $P_{\mathrm{r}}=3.8 \%$, which is a little smaller than the values obtained by the swelling method $(11 \%)^{18}$ and by the apparent density method $(3-7 \%) \cdot{ }^{18}$

\section{Appendix I. Derivations of eq 20 and 34}

Figure A-1(a) shows a schematic representation of a straight through cylindrical pore in a membrane, and Figure A-1(b) shows the flow lines of the solution on the membrane and in the pore. In Figure A-1(a), the center of the circular pore on the membrane surface is taken as the origin 0 in Cartesian coordinate $X, Y, Z$. The solution (filtrand) flows parallel to the $X$ axis from left to right. $d_{\mathrm{m}}$ and $d$ are the thicknesses of the membrane and the width of the gap between the membrane and a wall, respectively. In Figure A-1(b) is a flow partern in a cross section at $Z=z(x)$ parallel to the $X$ and $Y$ axes. Here, $x$ is the $X$-ordinate of this cross section

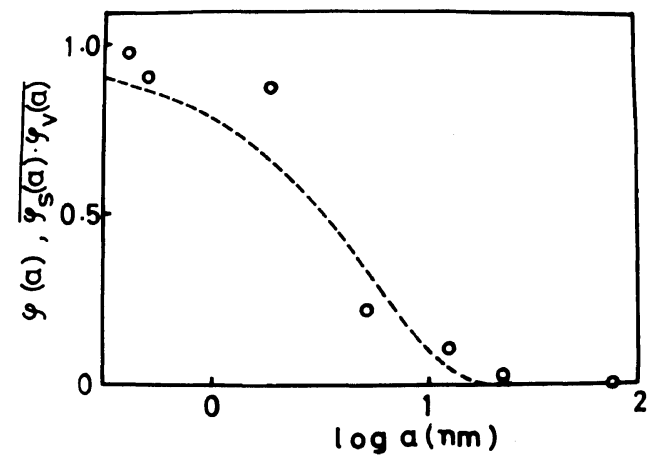

Figure 22. Observed $\varphi(a)$ of model substances with particle size $2 a$ at $\dot{\gamma}=0$ for Cuprophan: open circles, observed values and broken line, theoretical values $\overline{\varphi_{\mathrm{s}}(a) \cdot \varphi_{\mathrm{v}}(a)}$.

(c)

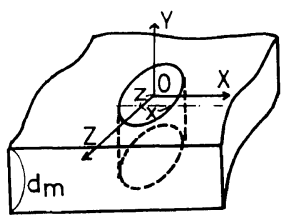

(b)

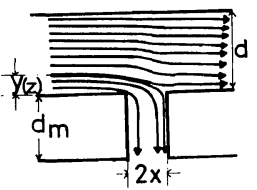

(c)

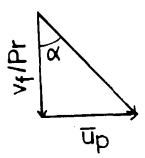

Figure A-1. Schematic representation of flow of filtrand above membrane surface: (a), Cartesian cordinate $X, Y$, and $Z ; d_{\mathrm{m}}$, thickness of the membrane; (b), flow line of filtrand and filtrate; $d$, slit width of filtrand flow; $2 x$, distance between pore walls in the direction of the $X$-axis at the point $Z=z ; y(z)$, thickness of laminar flow which can pass through a pore at $Z=z$; (c), definition of collision angle $\alpha ; v_{\mathrm{f}}$, filtration rate; $P_{\mathrm{r}}$, membrane porosity; $\bar{u}_{\mathrm{p}}$, mean velocity of the filtrand capable of passing through pore.

and is related to $z$ and $r$ by

$$
x^{2}+z^{2}=r^{2}
$$

where $r$ is pore radius. The function $x$ which satisfies eq A-1.1 is denoted by $x(z)$. We assume that the filtrand flow on the membrane is by a two dimen- 
sional Poiseuille flow and that when the flow rate is not zero, the component of the filtrand flowing into a pore from the $Z$ direction is negligible in its amount. From the material balance of the solvent between pore entrance and exit, we obtain

$$
\int_{0}^{y(z)} U_{\max }\left\{1-4(d / 2-y)^{2} / d^{2}\right\} \mathrm{d} y=\left(2 x(z) v_{\mathrm{f}} / P_{\mathrm{r}}\right)
$$

where $y(z)$ is the maximum value of $y$ for the flow which can enter a pore and depends on $z$ ( $c f$. Figure A-1(b)). $U_{\max }$ is the flow rate of the filtrand at $y=d / 2$. Since $y(z) \ll d$ holds generally, we obtain from eq A-1.2,

$$
y(z)=\left(x(z) v_{\mathrm{f}} \cdot d / U_{\max } P_{\mathrm{r}}\right)^{1 / 2}
$$

The average flow rate $\bar{u}_{\mathrm{p}}$ of the filtrand which can enter a pore $\bar{u}_{\mathrm{p}}$ is evaluated from,

$$
\bar{u}_{\mathrm{p}}=\int_{0}^{r} y(z) \bar{u}_{\mathrm{p}}(z) \mathrm{d} z / \int_{0}^{r} y(z) \mathrm{d} z
$$

with

$$
\begin{aligned}
\bar{u}_{\mathrm{p}}(z) & =\int_{0}^{y(z)} U_{\max }\left\{1-4(d / 2-y)^{2} / d\right\} \mathrm{d} y / \int_{0}^{y(z)} \mathrm{d} y \\
& =2 x v_{\mathrm{f}} / P_{\mathrm{r}} \cdot y(z)
\end{aligned}
$$

By substituting eq A-1.3 and A-1.5 into eq A-1.4, we obtain

$$
\bar{u}_{\mathrm{p}}=(1 / 2)\left(v_{\mathrm{f}} \cdot U_{\max } / P_{\mathrm{r}} \cdot d\right)^{1 / 2} 2 \pi r^{2} / \int_{0}^{r} x(z)^{1 / 2} \mathrm{~d} z
$$

The numerical calculation of

$$
\int_{0}^{1}\left(1-z^{2}\right)^{1 / 4} \mathrm{~d} z
$$

gives us the following approximate equation,

$$
\int_{0}^{r} x(z)^{1 / 2} \mathrm{~d} z=r^{3 / 2} \int_{0}^{1}(1-z / r) \mathrm{d}(z / r)=0.87 r^{3 / 2}
$$

Substitution of eq A-1.7 into eq A-1.6 gives

$$
\bar{u}_{\mathrm{p}}=0.575\left(r \cdot v_{\mathrm{f}} \cdot u_{\max } / P_{\mathrm{r}} \cdot d\right)^{1 / 2}
$$

The collision angle $\alpha$ is approximated by the angle whose tangent is the ratio between $\bar{u}_{\mathrm{p}}$ and $v_{\mathrm{f}} / P_{\mathrm{r}}$, as shown in Figure A-1(c). Thus, we get

$$
\alpha=\tan ^{-1}\left(\bar{u}_{\mathrm{p}} \cdot P_{\mathrm{r}} / v_{\mathrm{f}}\right)
$$

If $\bar{u}$ is defined as the mean velocity of the filtrand, assumption of a two dimensional Poiseuille flow for the filtrand leads to

$$
\bar{u}=(2 / 3) U_{\max }
$$

By substituting eq A-1.8 and A-1.10 into eq A-1.9, we obtain eq 20 in the text.

The mean value of $y(z), \bar{y}$, is defined by,

$$
\bar{y}=\int_{-r}^{r} y(z) \mathrm{d} z / \int_{-r}^{r} \mathrm{~d} z
$$

Unless $\bar{u}$ is 0 , i.e., if $U_{\max } \neq 0$, eq $\mathrm{A}-1.11$ is transformed to,

$$
\begin{aligned}
\bar{y}= & \int_{-r}^{r} y(z) \bar{u}_{\mathrm{p}}(z) \mathrm{d} z / \int_{-r}^{r} \mathrm{~d} z / \\
& \times \int_{-r}^{r} y(z) \bar{u}_{\mathrm{p}}(z) \mathrm{d} z / \int_{0}^{r} y(z) \mathrm{d} z
\end{aligned}
$$

If we substitute eq A-1.4 and A-1.6 into eq A-1.12, we obtain,

$$
\bar{y}=0.87\left(v_{\mathrm{f}} \cdot d \cdot r / P_{\mathrm{r}} \cdot U_{\max }\right)^{1 / 2}
$$

\section{Appendix II. Derivation of eq 42}

Figure A-2 shows a small volume element $\mathrm{d} x \cdot \mathrm{d} y \cdot \mathrm{d} z$ at the position $(x, y, z)$. The concentration of a particle has no gradient in either the $x$ or $z$ directions. Therefore, this concentration at the position $(x, y, z)$ can be expressed as $C(y)$. From the material balance, we obtain

$(\mathrm{d} C(y) / \mathrm{d} t) \mathrm{d} x \cdot \mathrm{d} y \cdot \mathrm{d} z=\left\{v_{(y-\mathrm{d} y / 2)}\right.$

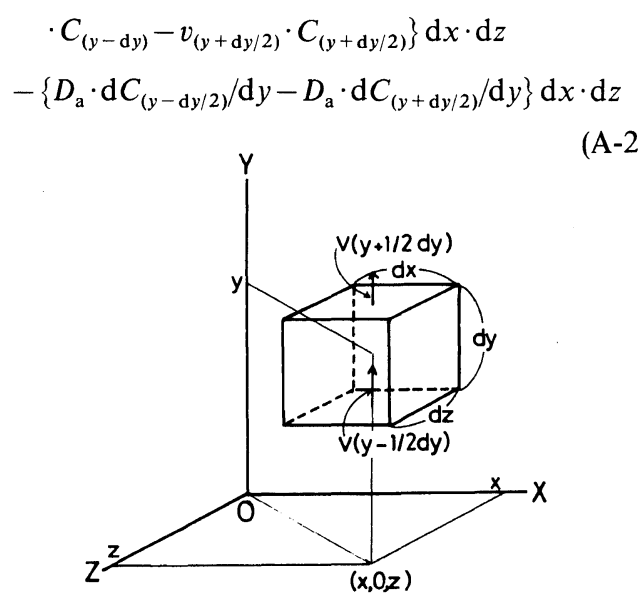

Figure A-2. Volume element the $\mathrm{d} x \cdot \mathrm{d} y \cdot \mathrm{d} z$ at point $(x, y, z)$ in the filtrand. 
where $D_{\mathrm{a}}$ is the diffusion coefficient of a solute in the solution. Equation A-2.1 can be rearranged to

$$
\begin{aligned}
\mathrm{d} C(y) / \mathrm{d} t= & -(\mathrm{d} C(y) / \mathrm{d} y) v(y)-(\mathrm{d} v(y) / \mathrm{d} y) C(y) \\
& +D_{\mathrm{a}}\left(\mathrm{d}^{2} C(y) / \mathrm{d} y^{2}\right)
\end{aligned}
$$

At the stationary state, the relation $\mathrm{d} C(y) / \mathrm{d} t=0$ holds, and eq A-2.2 becomes,

$$
\mathrm{d}^{2} C(y) / \mathrm{d} y^{2}=\left(1 / D_{\mathrm{a}}\right) \cdot \mathrm{d}(C(y) \cdot v(y)) / \mathrm{d} y
$$

The solution of eq A-2.3, by use of eq 39 , is given as,

$$
\begin{aligned}
\ln C(y)=- & \left(1 / 3 D_{\mathrm{a}}\right) B U_{\max }^{2}(2 a / d)^{2.84}(1-2 y / d)^{2} \\
& \times\{1-(\mathrm{d} / 3 R(a, d))(1-2 y / d)\} \\
& +C_{1} y+C_{2}
\end{aligned}
$$

where, $C_{1}$ and $\mathrm{C}_{2}$ are integration constants. By consideration of the boundary conditions, $\mathrm{d} C(y) / \mathrm{d} y=0$ at $v_{y}=0$ and that $C(y)=\bar{C}_{\mathrm{a}}$ at $y=d / 2$, eq A-2.4 can take the form of eq 42 in the text:

$$
\begin{aligned}
C(y)= & \bar{C}_{\mathrm{a}} \exp \left[-\left(B / 4 D_{\mathrm{a}}\right) \gamma^{2}(2 a / d)^{2.84}(1-2 y / d)\right. \\
& \times\{1-(d / 3 R(a, d))(1-\bar{y} / d)\}]
\end{aligned}
$$

Acknowledgement. This experiment was carried out in part at the Department of Urology, Osaka Medical College, Osaka, Japan, where the authors served as visiting lecturers. The authors should like to express their sincere gratitude to Drs. K. Sato, H. Okunishi and K. Hamada of Osaka Medical Colledge and Mr. T. Matsui of our laboratory for their helpful comments and skillful technical assistance.

\section{REFERENCES}

1. For example, C. P. Bean, Ed., "Membranes," Vol. 1, Mercel Dekker, New York, N.Y., 1972, Chapter 1.

2. For example, R. E. Kesting, "Synthetic Polymeric
Membranes," McGraw Hill, New York, N.Y., 1971.

3. J. P. Agrawal and S. Sourirajan, J. Appl. Polym. Sci., 14, 1303 (1970).

4. H. K. Lonsdale, U. Merten, and R. L. Riley, J. Appl. Polym. Sci., 9, 134 (1965).

5. J. D. Ferry, J. Gen. Physiol., 20, 95 (1936).

6. E. M. Renkin, J. Gen. Physiol., 38, 225 (1955).

7. K. Kamide, S. Manabe, K. Hamada, H. Okunishi, and S. Miyazaki, Artif. Organ. suppl., 5, 139 (1976).

8. K. Kamide and S. Manabe, "Ultrafiltration Membranes and Applications," A. R. Cooper, Ed., Plenum Publishing Corp, New York, 1980, p 173.

9. K. Kamide, S. Manabe, K. Sato, K. Hamada, H. Okunishi, and S. Miyazaki, Artif. Organ, 4, 171 (1975).

10. For example, A. Katchalsky and P. F. Curran, "Nonequilibrium Thermodynamics in Biophysics," Harvard University Press, Cambridge, 1967, Chapter 10.

11. H. Faxén, Kolloid. Z., 167, 146 (1959).

12. J. Happel and B. J. Byrne, Ind. Eng. Chem., 46, 1181 (1954).

13. H. L. Goldsmith and S. G. Mason, J. Colloid Sci., 17, 448 (1962).

14. H. P. Schreiber and S. H. Storey, J. Polym. Sci., B, 3, 723 (1965).

15. For example, H. L. Goldsmith and S. G. Mason, "Rheology," Vol. 4, F. R. Eirich, Ed., Academic Press, New York and London, 1967, Chapter 2, p 217.

16. For example, B. Chu, "Molecular Force: Based on the Baker Lectures of Peter J. W. Debye," John Willey \& Sons, New York, N.Y., 1967.

17. K. Kamide, S. Manabe, T. Matsui, T. Sakamoto, and S. Kajita, Kobunshi Ronbunshu, 34, 205 (1977).

18. K. Kamide, S. Manabe, and T. Matsui, Kobunshi Ronbunshu, 34, 299 (1977).

19. For example, R. L. Goldsmith, Ind. Eng. Chem. Fundam., 10, 113 (1971).

20. For example, W. R. Krigbaum, J. Polym. Sci., 28, 213 (1958).

21. For example, S. Bottomley, F. M. Parsons, and P. M. G. Broughton, J. Appl. Polym. Sci., 16, 2115 (1972). 